\title{
Evaluation of Effective Elastic, Piezoelectric, and Dielectric Properties of SU8/ZnO Nanocomposite for Vertically Integrated Nanogenerators Using Finite Element Method
}

\author{
Neelam Mishra, Braj Krishna, Randhir Singh, and Kaushik Das \\ School of Minerals, Metallurgical and Materials Engineering, Indian Institute of Technology Bhubaneswar, \\ Toshali Bhawan, Bhubaneswar, Odisha 751007, India
}

Correspondence should be addressed to Kaushik Das; kaushik@iitbbs.ac.in

Received 24 January 2017; Accepted 13 April 2017; Published 15 May 2017

Academic Editor: R. Torrecillas

Copyright (C) 2017 Neelam Mishra et al. This is an open access article distributed under the Creative Commons Attribution License, which permits unrestricted use, distribution, and reproduction in any medium, provided the original work is properly cited.

\begin{abstract}
A nanogenerator is a nanodevice which converts ambient mechanical energy into electrical energy. A piezoelectric nanocomposite, composed of vertical arrays of piezoelectric zinc oxide $(\mathrm{ZnO})$ nanowires, encapsulated in a compliant polymeric matrix, is one of most common configurations of a nanogenerator. Knowledge of the effective elastic, piezoelectric, and dielectric material properties of the piezoelectric nanocomposite is critical in the design of a nanogenerator. In this work, the effective material properties of a unidirectional, unimodal, continuous piezoelectric composite, consisting of SU8 photoresist as matrix and vertical array of ZnO nanowires as reinforcement, are systematically evaluated using finite element method (FEM). The FEM simulations were carried out on cubic representative volume elements (RVEs). Four different types of arrangements of $\mathrm{ZnO}$ nanowires and three sizes of RVEs have been considered. The volume fraction of $\mathrm{ZnO}$ nanowires is varied from 0 to a maximum of 0.7. Homogeneous displacement and electric potential are prescribed as boundary conditions. The material properties are evaluated as functions of reinforcement volume fraction. The values obtained through FEM simulations are compared with the results obtained via the Eshelby-MoriTanaka micromechanics. The results demonstrate the significant effects of $\mathrm{ZnO}$ arrangement, $\mathrm{ZnO}$ volume fraction, and size of RVE on the material properties.
\end{abstract}

\section{Introduction}

Mechanical energy is one of the most ubiquitous and abundant among sources of energy. Mechanical energy can be harvested from wind flows [1], from ambient vibrations [2], and from the human movements [3]. Advanced piezoelectric materials, especially piezoelectric composites, are central in harvesting mechanical energy and converting it into electrical energy. The mechanical strain in these material leads to the development of electric potential which can be used to charge a battery in portable electronic devices or be used for stand-alone, self-powered nanodevices [4]. A mechanical energy-harvesting nanodevice based on piezoelectric zinc oxide $(\mathrm{ZnO})$ nanowires was first demonstrated by Wang and Song in 2006 [5]. Since then, a new group of energyharvesting nanosystem, known as nanogenerator, has been developed [6-9]. The most common configuration of a nanogenerator involves piezoelectric $\mathrm{ZnO}$ nanowire grown vertically on a silicon substrate [10]. The vertically grown array of $\mathrm{ZnO}$ nanowires is encapsulated with a polymer, which may be active or passive [11]. Combination of photopatternable polymer SU8 with $\mathrm{ZnO}$ nanowires is expected to create a nanocomposite that can be easily integrated with the microfabrication process flow established for fabrication of microsystems and flexible electronics. Design and optimization of a nanogenerator with nanocomposites as the active material require knowledge of the effective elastic, piezoelectric, and dielectric properties of the nanocomposite.

Prediction of effective properties of a piezoelectric composite can be accomplished by numerous approaches. Simple Voigt and Reuss approaches [12], developed for uncoupled elastic composites, have been extended to predict upper 
and lower bounds of effective dielectric and piezoelectric constants of composites with specific reinforcement arrangements $[13,14]$. Bisegna and Luciano have extended HashinShtrikman type of variational approaches [15] to electroelastic problems and have estimated bounds of effective material properties $[14,16]$. Several authors have extended Eshelby's classical solution [17] of an ellipsoidal inclusion in a homogeneous matrix to the field of linear piezoelectricity and have calculated the effective electroelastic constants [18-20]. Odegard has proposed a new model by generalizing the MoriTanaka [21, 22] and self-consistent [23, 24] approaches, for predicting the coupled electromechanical properties of piezoelectric composites [25]. Using asymptotic homogenization method Guinovart-Díaz et al. have derived exact expressions for binary piezoelectric composites where both components have $6 \mathrm{~mm}$ symmetry for cases where the reinforcement is a piezoelectric ceramic and the polymeric matrix may or may not be piezoelectrically active [26].

In addition to these analytical and semianalytical approaches, computational methods based on finite element modeling have also been employed to evaluate effective properties of piezoelectric composites. The approaches based on FEM are extensions of the representative volume element (RVE) approach commonly employed to solve problems of linear elasticity in case of composites and foams $[27,28]$. Gaudenzi employed a RVE-based FEM to evaluate the electromechanical response of a $0-3$ piezoelectric composite [29]. Poizat and Sester have evaluated effective longitudinal and transverse piezoelectric constants of $0-3$ and 1-3 composites with embedded piezoelectric fibres using FEM [30]. Pettermann and Suresh have developed a RVEbased model and predicted the complete set of moduli for piezoelectric composites with periodic hexagonal and square array of continuous fibres [31]. The effect of fibre distribution, effect of fibre shape and geometric connectivity, and effect of poling on the effective electromechanical properties of 1-3 piezoelectric composites have been reported by Kar-Gupta and Venkatesh [32-34]. Unit cell models of unidirectional periodic 1-3 piezoelectric composites using periodic boundary conditions have also been reported by Berger et al. [35, 36] and by Medeiros et al. [37]. Recent years have seen an interest in nonlinear finite element modeling of laminated piezoelectric composites [38, 39].

Literature review revealed a gap in the experimental as well computational study of process-structure-property relationships of nanocomposites for applications in nanogenerators. Previous work on nanogenerators with piezoelectric $\mathrm{ZnO}$ nanowires embedded in a PMMA matrix used simplistic Reuss and Voigt analyses to predict effective properties of the composite, assuming both the matrix and reinforcement to be isotropic [8]. This work presents a rigorous and systematic analysis based on FEM which can be applied for piezoelectric nanocomposites with anisotropic constituents, as well as with different geometries of the reinforcement phase, as long as the RVE has three mutually perpendicular planes of mirror symmetry passing through the centroid of the RVE. To the best of our knowledge, this work represents the first systematic study of the effect of $\mathrm{ZnO}$ nanowire volume fraction and $\mathrm{ZnO}$ nanowire arrangement on the effective elastic, piezoelectric, and dielectric properties of a unidirectional, unimodal SU8/ZnO nanocomposites. The effective properties of the SU8/ZnO nanocomposite were obtained by FEM and by an approach based on Eshelby-MoriTanaka (EMT) micromechanics.

The article is arranged in the following fashion. First, the linear theory of piezoelectricity is introduced in Section 2. Next, two approaches of prediction of effective properties of a piezoelectric composite are discussed. Section 2.1 discusses the approach based on EMT micromechanics, while Section 2.2 presents the FEM study on idealised RVE. Section 3 presents the different types of RVEs considered in this study. The material properties of the constituent phases are provided in Section 4. The boundary conditions applied to the RVEs to calculate the effective material properties are presented in Section 5. Finally, the results of the finite element analyses and EMT micromechanics-based approach are presented and compared in Section 6. The results are discussed in Section 7 and final conclusions are drawn in Section 8.

\section{Theory}

The theory of linear piezoelectricity couples the interaction between the mechanical (elastic) and electric variables by the following constitutive equations [40]:

$$
\begin{aligned}
\sigma_{i j} & =C_{i j k l} \varepsilon_{k l}-e_{l i j} E_{\mathbf{l}}, \\
D_{\mathbf{i}} & =e_{i k l} \varepsilon_{k l}+\kappa_{i k} E_{k},
\end{aligned}
$$

where the indices $i, j, k$, and $l$ range from 1 to 3 . Here, $\varepsilon_{k l}$ are the components of the elastic strain, $E_{1}$ are the components of the electric field, $\sigma_{i j}$ are the components of the stress tensor, and $D_{\mathbf{i}}$ are the components of the electric displacement. In this stress-charge form of piezoelectric constitutive equations, the elastic strain tensor and the electric field vector are the independent variables. $C_{i j k l}$ are components of the fourthorder elastic stiffness tensor measured in the absence of an applied electric field. $e_{l i j}$ are components of the piezoelectric modulus tensor measured in the absence of an applied strain. $\kappa_{i k}$ are components of the dielectric modulus measured in the absence of an applied strain. In addition, solution of any problem in the domain of linear piezoelectricity requires that the equations of elastic equilibrium and Gauss' law of electrostatics be satisfied. In the absence of body forces, the equation for elastic equilibrium is given by

$$
\sigma_{i j, j}=0 .
$$

In (2) and in the following, the Einstein summation convention is used. The comma signifies partial differentiation with respect to the coordinate following it. The Gauss equation of electrostatics in the absence of free charges can be expressed as

$$
D_{i, i}=0 .
$$

The components of the strain tensor, $\varepsilon_{i j}$, and the components of the electric field, $E_{i}$, are related to displacement $\left(u_{i}\right)$ and electric potential $(\varphi)$ by the following equations:

$$
\begin{aligned}
\varepsilon_{i j} & =\frac{1}{2}\left(u_{i, j}+u_{j, i}\right), \\
E_{i} & =-\varphi_{, i} .
\end{aligned}
$$


In modeling of piezoelectric behaviour, it is convenient to treat the elastic and the electric variables in a similar fashion. This is accomplished by employing a notation introduced by Barnett and Lothe [41], which involves uppercase indices that range from 1 to 4 , as well as lowercase indices that range from 1 to 3 . According to this notation, the stress and electric displacement are represented by

$$
\Theta_{i J}= \begin{cases}\sigma_{i j} & \text { for } J=1,2,3 \\ D_{i} & \text { for } J=4 .\end{cases}
$$

And, the elastic strain and the electric field are represented by

$$
Z_{K l}= \begin{cases}\varepsilon_{k l} & \text { for } K=1,2,3 \\ -\phi_{, l} & \text { for } K=4 .\end{cases}
$$

The electroelastic moduli relating $\Theta_{i J}$ and $Z_{K l}$ can be represented as

$$
\Sigma_{i J K l}= \begin{cases}C_{i j k l} & \text { for } J=1,2,3 ; K=1,2,3, \\ e_{l i j} & \text { for } J=1,2,3 ; K=4, \\ e_{i k l} & \text { for } J=4 ; K=1,2,3, \\ -\kappa_{i l} & \text { for } J=4 ; K=4 .\end{cases}
$$

In short-hand notation,

$$
\Theta_{i J}=\Sigma_{i J K l} Z_{K l} .
$$

It becomes convenient to represent the constitutive equations in a generalized Voigt two-index notation (with two indices, each ranging from 1 to 9) as $[19,42]$. The generalized Voigt notation involves first representing $\Theta_{i J}$ and $Z_{K l}$ as $9 \times 1$ matrices and transforming the $(i J K l)$ index of the electroelastic moduli to a two-index form. The following transformation of subscripts is as follows:

$$
\begin{array}{r}
(11) \longrightarrow 1 ; \\
(22) \longrightarrow 2 ; \\
(33) \longrightarrow 3 ; \\
(23),(32) \longrightarrow 4 ; \\
(13),(31) \longrightarrow 5 ; \\
(12),(21) \longrightarrow 6 ; \\
(14),(41) \longrightarrow 7 ; \\
(24),(42) \longrightarrow 8 ; \\
(34),(43) \longrightarrow 9
\end{array}
$$

Thus, $\Sigma_{1122}$ would transform to $\Sigma_{12}$ with a value of $C_{12}$, while $\Sigma_{3443}$ would transform to $\Sigma_{99}$ with a value of $-\kappa_{33}$. Therefore, the constitutive equations can now be represented as

$$
\left[\begin{array}{l}
\sigma \\
\mathrm{D}
\end{array}\right]=\left[\begin{array}{cc}
\mathrm{C} & \mathrm{e}^{\mathrm{T}} \\
\mathrm{e} & -\kappa
\end{array}\right]\left[\begin{array}{c}
\varepsilon \\
-\mathrm{E}
\end{array}\right] .
$$

The electroelastic modulus matrix $(\boldsymbol{\Sigma})$ can now be defined as

$$
\Sigma=\left[\begin{array}{ll}
\mathrm{C} & \mathrm{e}^{\mathrm{T}} \\
\mathrm{e} & -\boldsymbol{\kappa}
\end{array}\right]
$$

For an isotropic nonpiezoelectric solid, (i) there are two nonzero, independent constants for the stiffness matrix, that is, $C_{11}$ and $C_{12}$, (ii) all components of the piezoelectric modulus matrix of this type of solid are zero, and (iii) there is only one independent constant for the dielectric modulus matrix. The electroelastic modulus for an isotropic passive matrix phase is given by

$$
\begin{aligned}
& \Sigma_{\mathbf{m}} \\
& =\left[\begin{array}{ccccccccc}
C_{11} & C_{12} & C_{12} & 0 & 0 & 0 & 0 & 0 & 0 \\
C_{12} & C_{11} & C_{12} & 0 & 0 & 0 & 0 & 0 & 0 \\
C_{12} & C_{12} & C_{11} & 0 & 0 & 0 & 0 & 0 & 0 \\
0 & 0 & 0 & C_{44} & 0 & 0 & 0 & 0 & 0 \\
0 & 0 & 0 & 0 & C_{44} & 0 & 0 & 0 & 0 \\
0 & 0 & 0 & 0 & 0 & C_{44} & 0 & 0 & 0 \\
0 & 0 & 0 & 0 & 0 & 0 & -\kappa_{11} & 0 & 0 \\
0 & 0 & 0 & 0 & 0 & 0 & 0 & -\kappa_{11} & 0 \\
0 & 0 & 0 & 0 & 0 & 0 & 0 & 0 & -\kappa_{11}
\end{array}\right] .
\end{aligned}
$$

For a transversely isotropic piezoelectric solid, where 1-2 plane is the plane of isotropy, there are (i) five independent constants for the stiffness matrix, that is, $C_{11}, C_{12}, C_{13}, C_{33}$, and $C_{44}$, (ii) three independent constants for the piezoelectric modulus, that is, $e_{13}, e_{15}$, and $e_{33}$, and (iii) two independent constants for the dielectric modulus, that is, $\kappa_{11}$ and $\kappa_{33}$. The electroelastic modulus for the reinforcement phase is given by

$$
\begin{aligned}
& \Sigma_{\mathbf{f}} \\
& =\left[\begin{array}{ccccccccc}
C_{11} & C_{12} & C_{13} & 0 & 0 & 0 & 0 & 0 & e_{13} \\
C_{12} & C_{11} & C_{13} & 0 & 0 & 0 & 0 & 0 & e_{13} \\
C_{13} & C_{13} & C_{33} & 0 & 0 & 0 & 0 & 0 & e_{33} \\
0 & 0 & 0 & C_{44} & 0 & 0 & 0 & e_{15} & 0 \\
0 & 0 & 0 & 0 & C_{44} & 0 & e_{15} & 0 & 0 \\
0 & 0 & 0 & 0 & 0 & C_{66} & 0 & 0 & 0 \\
0 & 0 & 0 & 0 & e_{15} & 0 & -\kappa_{11} & 0 & 0 \\
0 & 0 & 0 & e_{15} & 0 & 0 & 0 & -\kappa_{11} & 0 \\
e_{13} & e_{13} & e_{33} & 0 & 0 & 0 & 0 & 0 & -\kappa_{33}
\end{array}\right] .
\end{aligned}
$$

For a 1-3 piezoelectric composite, composed of an isotropic nonpiezoelectric matrix and with transversely isotropic piezoelectric reinforcements, the effective values of the components of the electroelastic matrix can be evaluated using (i) a micromechanics-based semianalytical approach and (ii) finite element method, as discussed in detail in the following subsections. 
2.1. Micromechanics-Based Approach. The effective electroelastic modulus of a two-phase piezoelectric composite can be expressed in terms of the electroelastic moduli and the volume fractions of the constituent phases. Under homogeneous elastic displacement and electric potential boundary conditions, generalizing the average strain theorem of elasticity [43] for piezoelectricity, the effective electroelastic modulus of a piezoelectric composite is obtained as follows [19]:

$$
\boldsymbol{\Sigma}=\boldsymbol{\Sigma}_{m}+v_{f}\left(\boldsymbol{\Sigma}_{f}-\boldsymbol{\Sigma}_{m}\right) \mathbf{A}
$$

Here, the subscripts $m$ and $f$ refer to the matrix and the reinforcement, respectively. $v_{f}$ is the volume fraction of the reinforcement, and $\mathbf{A}$ is the strain-potential gradient concentration matrix. A relates the average strain and potential gradient in the reinforcement phase to the applied homogeneous displacement and electric potential in the composite. Estimation of the concentration tensor $\mathbf{A}$ can be achieved through several micromechanical schemes, for example, by using the dilute approximation scheme [17], the self-consistent scheme [23], the differential scheme [24], and the EMT mean field approach [21, 22]. In this work, we have considered EMT mean field approach, which will be discussed in detail.

Consider the case of an ellipsoidal inclusion in an infinite matrix wherein the electroelastic fields of the reinforcement phases do not interact. The electromechanical response of such "dilute" composites can be obtained using the Eshelby method, which is also known as the dilute approximation method. For dilute approximation, the concentration tensor, $\mathrm{A}^{\text {dil }}$, is given by

$$
\mathbf{A}^{\mathrm{dil}}=\left[\mathbf{I}+\mathbf{S} \Sigma_{\mathbf{m}}^{-1}\left(\Sigma_{\mathrm{f}}-\boldsymbol{\Sigma}_{\mathbf{m}}\right)\right]^{-1}
$$

where $\mathbf{I}$ is the $9 \times 9$ identity matrix. $\mathbf{S}$ is analogous to Eshelby tensor [17] and is represented by a $9 \times 9$ matrix. Similar to Eshelby tensor, components of $\mathbf{S}$, also known as the constraint tensor, are functions of the geometry of the reinforcement phase and the electroelastic moduli of the matrix phase. Explicit expressions of $\mathbf{S}$ are available in the literature [18, 19, 44]. Now, for the nondilute approximation, when there are multiple inclusions present in the matrix, the electroelastic fields of the inclusions interact. Mori-Tanaka approach takes these interactions into account by modeling the interacting electroelastic field as a background electroelastic field. The modified concentration factor can be expressed as follows [22]:

$$
\mathbf{A}^{\mathbf{M T}}=\mathbf{A}^{\mathbf{d i l}}\left[\left(1-v_{f}\right) \mathbf{I}+v_{f} \mathbf{A}^{\mathbf{d i l}}\right]^{-1} .
$$

Combining (14) and (16), the effective electroelastic modulus of a piezoelectric composite can be estimated as follows:

$$
\Sigma=\Sigma_{m}+v_{f}\left(\Sigma_{f}-\Sigma_{m}\right) \mathbf{A}^{\mathrm{MT}} .
$$

The inverse of the effective electroelastic modulus matrix is represented by $\mathbf{F}$, that is,

$$
\mathbf{F}=\Sigma^{-1}
$$

The components of the piezoelectric strain coefficients matrix are defined as follows:

$$
\begin{aligned}
& d_{31}=-\frac{F_{91}}{F_{99}}, \\
& d_{33}=-\frac{F_{93}}{F_{99}}, \\
& d_{15}=-\frac{F_{75}}{F_{77}} .
\end{aligned}
$$

2.2. FEM Approach. The first step towards prediction of effective properties of a composite using FEM is to select an RVE or a unit cell. The RVE must be small enough compared to the macroscopic body, but it should be large enough to capture the major features of the microstructure [37]. An RVE may be of several geometries, for example, cubic, hexagonal, and spherical. A cubic RVE, in spite of several deficiencies like accommodation of low volume fraction of reinforcement, is most commonly used due to the relative ease of applying boundary conditions. A cubic RVE can be further reduced in size by taking into account the symmetry in the arrangement of the reinforcement phase(s). The micromechanics-based method, discussed in the previous section, assumes the stress and strain fields inside the inclusions to be constant, whereas finite element approach predicts a more-realistic picture of nonuniform stress and strain fields at each nodal point. The evaluation of the effective properties of a composite is then accomplished by employing the homogenization method. This method relates the volume average stress, strain, electric displacement, and electric field to the effective properties of the composite. The composite is thus modeled as a homogenized medium. Using FEM, the volume averages can be calculated as follows:

$$
\begin{aligned}
& \overline{\sigma_{i j}}=\frac{1}{V} \int \sigma_{i j} d V=\frac{1}{V} \sum_{n=1}^{\text {nel }} \sigma_{i j}^{(n)} V^{(n)} \\
& \overline{\varepsilon_{i j}}=\frac{1}{V} \int \varepsilon_{i j} d V=\frac{1}{V} \sum_{n=1}^{\text {nel }} \varepsilon_{i j}^{(n)} V^{(n)} \\
& \overline{D_{i}}=\frac{1}{V} \int D_{i} d V=\frac{1}{V} \sum_{n=1}^{\text {nel }} D_{i}^{(n)} V^{(n)} \\
& \overline{E_{i}}=\frac{1}{V} \int E_{i} d V=\frac{1}{V} \sum_{n=1}^{\text {nel }} E_{i}^{(n)} V^{(n)}
\end{aligned}
$$

In (20), $V$ is the volume of the RVE. $\overline{\sigma_{i j}}, \overline{\varepsilon_{i j}}, \overline{D_{i}}$, and $\overline{E_{i}}$ are the volume-averaged values of stress, strain, electric displacement, and electric field, respectively. nel is total number of finite elements in the RVE, $V^{(n)}$ is the volume of the $n$th element, $\sigma_{i j}^{(n)}$ is $i j$ th component of the stress tensor calculated in the $n$th element, and $\varepsilon_{i j}^{(n)}$ is $i j$ th component of the strain tensor calculated in the $n$th element. Similarly, $D_{i}^{(n)}$ and $E_{i}^{(n)}$ are the $i$ th component of the electric displacement field and of the electric field, respectively, each calculated 
TABLE 1: Characteristics of the RVEs with regard to their size and the density and arrangement of ZnO nanowires.

\begin{tabular}{lcccc}
\hline RVE name & $\begin{array}{c}\text { Arrangement of nanowires } \\
\text { in } x-y \text { plane }\end{array}$ & $\begin{array}{c}\text { Edge length of full cubic } \\
\text { RVE }(2 h)\end{array}$ & $\begin{array}{c}\text { Edge length of }(1 / 8) \text { th of the } \\
\text { full RVE }(h)\end{array}$ & $\begin{array}{c}\text { Effective number of nanowires } \\
\text { in full RVE }\end{array}$ \\
\hline RVE1 & Square array & $120 \mathrm{~nm}$ & $60 \mathrm{~nm}$ & 1 \\
RVE2 & Square array & $240 \mathrm{~nm}$ & $120 \mathrm{~nm}$ & 4 \\
RVE3 & Face-centred rectangle array & $240 \mathrm{~nm}$ & $120 \mathrm{~nm}$ & 4 \\
RVE4 & Face-centred square array & $240 \mathrm{~nm}$ & $120 \mathrm{~nm}$ & 8 \\
RVE5 & Face-centred square array & $120 \mathrm{~nm}$ & $60 \mathrm{~nm}$ & 2 \\
RVE6 & Quasi-random & $800 \mathrm{~nm}$ & $400 \mathrm{~nm}$ & 64 \\
\hline
\end{tabular}

in the $n$th element. Consider a unidirectional composite, where the matrix is passive and isotropic and the piezoelectric reinforcement is transversely isotropic. The local 3-axis of the piezoelectric reinforcement is aligned with the global $z$-axis. In terms of these average values, discussed earlier, the constitutive equations of linear piezoelectricity for this material can be expressed in the matrix form as follows:

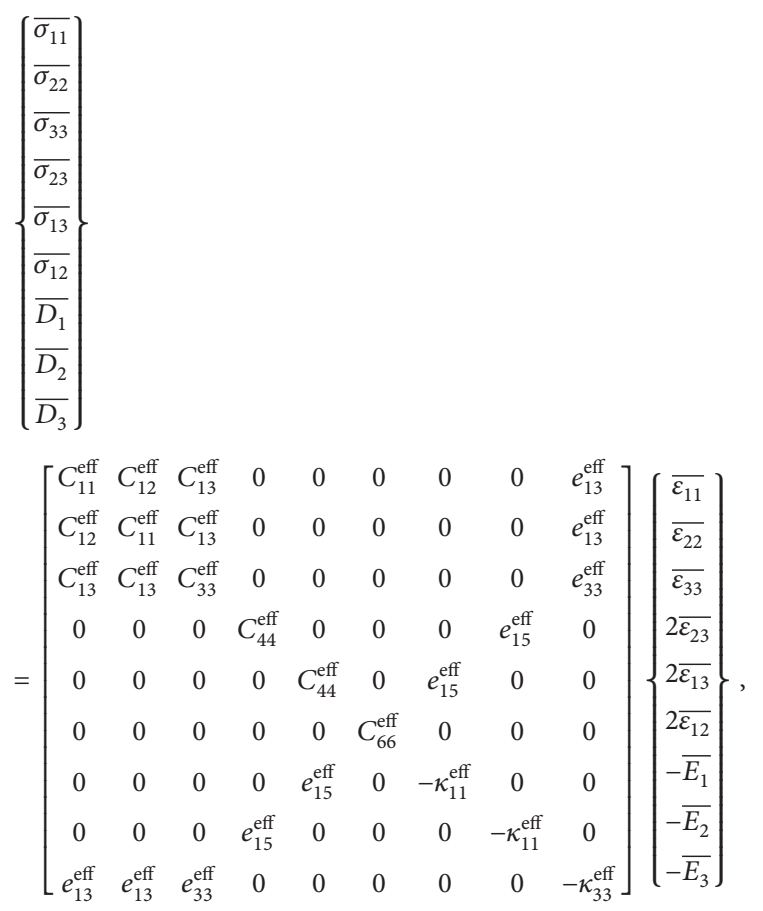

where the superscript eff refers to the effective properties of the composite. Equation (21) represents the basis on which the boundary conditions are applied on the RVE. For homogeneous applied strain $\left(\varepsilon_{i j}^{0}\right)$ and homogeneous applied electric field $\left(E_{i}^{0}\right)$, the boundary conditions applied on the surfaces of the cubic RVE are of the following form:

$$
\begin{aligned}
& u_{i}=\varepsilon_{i j}^{0} x_{j}, \\
& \varphi=-E_{i}^{0} x_{i},
\end{aligned}
$$

where $x_{i}$ refers to the coordinates of the surfaces of the RVE.

\section{Types of RVE}

The RVEs considered are cubes of fixed dimensions $(2 h \times 2 h \times$ $2 h$ ) with centroid at the origin and with the boundaries given by the following:

$$
\begin{aligned}
& S_{1}: x=-h,-h \leq y \leq h,-h \leq z \leq h, n=(-1,0,0) \\
& S_{2}: x=+h,-h \leq y \leq h,-h \leq z \leq h, n=(1,0,0) \\
& S_{3}: y=-h,-h \leq x \leq h,-h \leq z \leq h, n=(0,-1,0) \\
& S_{4}: y=+h,-h \leq x \leq h,-h \leq z \leq h, n=(0,1,0) \\
& S_{5}: z=-h,-h \leq x \leq h,-h \leq y \leq h, n=(0,0,-1) \\
& S_{6}: z=+h,-h \leq x \leq h,-h \leq y \leq h, n=(0,0,1)
\end{aligned}
$$

$S_{1}-S_{6}$ are the six bounding surfaces of the representative cubic volume element. The nanowires are oriented along the $z$-direction. The RVEs are chosen such that reflectional symmetries are present about $x$-, $y$-, and $z$-axes, and hence only $(1 / 8)$ th of the cubes have been considered for finite element analysis. The boundaries $\left(B_{1}-B_{6}\right)$ of the reduced structures are as follows:

$$
\begin{aligned}
& B_{1}: x=0,0 \leq y \leq h, 0 \leq z \leq h, \mathbf{n}=(-1,0,0) \\
& B_{2}: x=+h, 0 \leq y \leq h, 0 \leq z \leq h, \mathbf{n}=(1,0,0) \\
& B_{3}: y=0,0 \leq x \leq h, 0 \leq z \leq h, \mathbf{n}=(0,-1,0) \\
& B_{4}: y=+h, 0 \leq x \leq h, 0 \leq z \leq h, \mathbf{n}=(0,1,0) \\
& B_{5}: z=0,0 \leq x \leq h, 0 \leq y \leq h, \mathbf{n}=(0,0,-1) \\
& B_{6}: z=+h, 0 \leq x \leq h, 0 \leq y \leq h, \mathbf{n}=(0,0,1)
\end{aligned}
$$

Here, $\mathbf{n}$ represents the outward unit normal vector to the bounding surface. Six types of cubic RVEs are considered in this study. The RVEs differ from one another in terms of size of the unit cube and the arrangement of the nanowires on the $x-y$ plane. The sizes of the RVEs are chosen such that the maximum diameter of the $\mathrm{ZnO}$ nanowires, corresponding to the maximum volume fraction for each RVE, is in the range of $80-110 \mathrm{~nm}$. Three different sizes and four different arrangements are considered, with an aim to study the effect of RVE-size as well as the effect of the distribution of the $\mathrm{ZnO}$ nanowires on the effective properties of the nanocomposite. Details of these RVEs are provided in Table 1. RVE1 and RVE2 both have the identical square array in terms of distribution of the nanowires on the $x-y$ plane. Images of RVE1 and RVE2 are provided in Figures $1(\mathrm{a})$ and $1(\mathrm{~b})$, respectively. In RVE3, the nanowires are distributed in a face-centred 


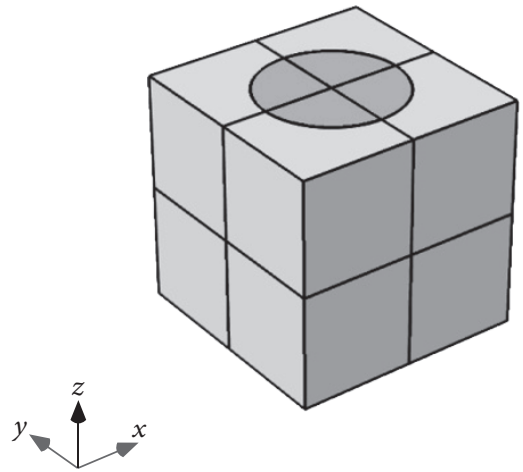

(a)

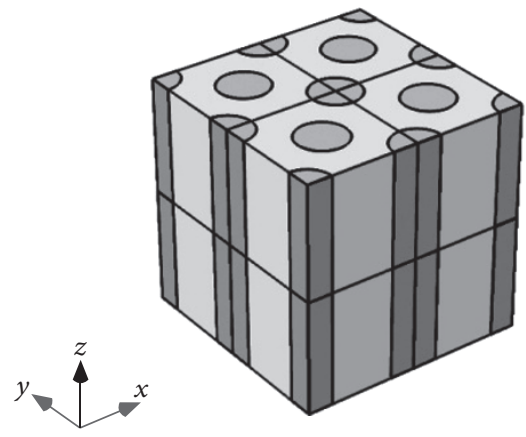

(d)
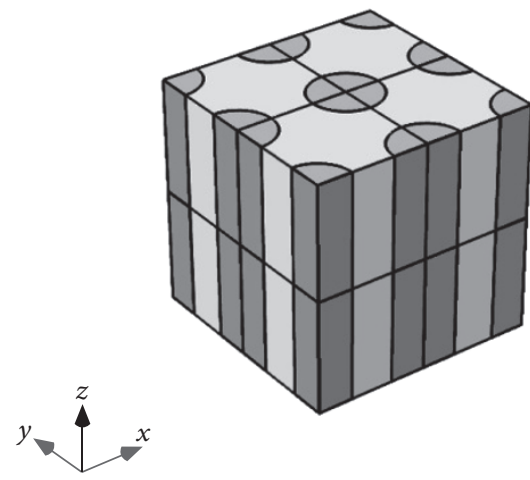

(b)
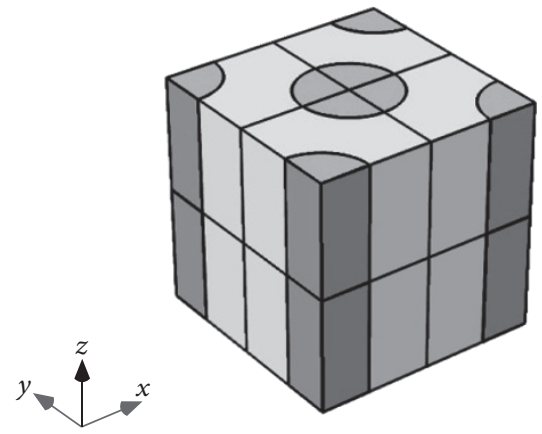

(e)
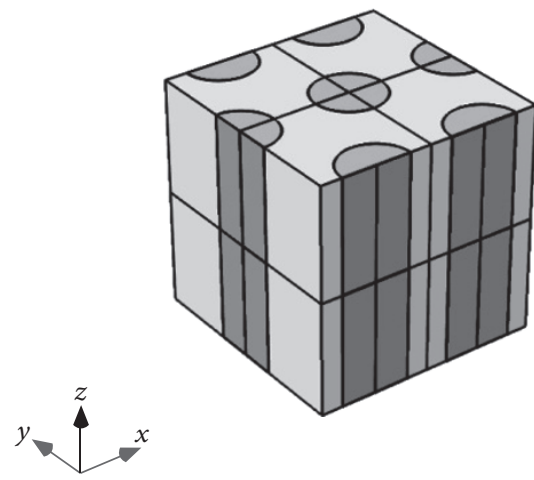

(c)
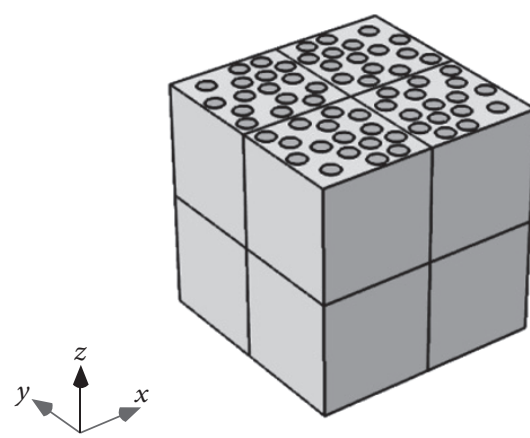

(f)

FIGURE 1: Images of full RVEs showing (a) RVE1 with cube-edge length of $120 \mathrm{~nm}$, (b) RVE2 with cube-edge length of $240 \mathrm{~nm}$, (c) RVE3 with cube-edge length of $240 \mathrm{~nm}$, (d) RVE4 with cube-edge length of $240 \mathrm{~nm}$, (e) RVE5 with cube-edge length of $120 \mathrm{~nm}$, and (f) RVE6 with cube-edge length of $800 \mathrm{~nm}$. The lighter regions represent the matrix, whereas the darker regions represent the cylindrical $\mathrm{ZnO}$ nanowires. The traces of the mirror planes, that is, $x=0, y=0$, and $z=0$, are also shown.

rectangular array, as shown in Figure 1(c). In RVE4 and in RVE5, the nanowires are distributed in a face-centred square array, as shown in Figures 1(d) and 1(e), respectively. In RVE1-RVE5, the maximum reinforcement volume fraction considered is 0.7 since the maximum theoretical volume fraction of cylindrical reinforcements in a cubic volume is 0.785. In RVE6, as shown in Figure 1(f), the nanowires are distributed in a quasi-random fashion, while maintaining the reflectional symmetries. The maximum volume fraction of the reinforcement in RVE6 is limited to $\sim 0.52$ to ensure that the fibre geometries do not intersect each other.

\section{Material Properties}

The matrix, SU8, is a negative photoresist, commonly employed in microfabrication, and nanocomposites with SU8 as matrix have been used to fabricate structural components of MEMS sensors [48-52]. The mechanical properties of this polymer depend on the processing conditions. The material properties of SU8 considered in this study assume that the photoresist has been hard-baked at $\sim 95^{\circ} \mathrm{C}$. SU8 is considered as isotropic in terms of elastic and dielectric properties and it does not exhibit piezoelectricity. The reinforcement $\mathrm{ZnO}$ is assumed to have a wurtzite structure, which is transversely isotropic. The elastic, piezoelectric, and dielectric constants of the electroelastic moduli of the matrix and the reinforcement are provided in Table 2 .
TABLE 2: Material properties of SU8 $[45,46]$ and $\mathrm{ZnO}[47]$.

\begin{tabular}{lccc}
\hline Property & Units & SU8 & $\mathrm{ZnO}$ \\
\hline$C_{11}$ & $\mathrm{GPa}$ & 5.02 & 209.71 \\
$C_{12}$ & $\mathrm{GPa}$ & 1.42 & 121.14 \\
$C_{13}$ & $\mathrm{GPa}$ & 1.42 & 105.36 \\
$C_{33}$ & $\mathrm{GPa}$ & 5.02 & 211.19 \\
$C_{44}$ & $\mathrm{GPa}$ & 1.80 & 42.37 \\
$C_{66}$ & $\mathrm{GPa}$ & 1.80 & 44.28 \\
$e_{13}$ & $\mathrm{C} / \mathrm{m}^{2}$ & 0 & -0.567005 \\
$e_{33}$ & $\mathrm{C} / \mathrm{m}^{2}$ & 0 & 1.32044 \\
$e_{15}$ & $\mathrm{C} / \mathrm{m}^{2}$ & 0 & -0.480508 \\
$\kappa_{11}$ & $\mathrm{nF} / \mathrm{m}$ & 0.0354 & 0.0757 \\
$\kappa_{33}$ & $\mathrm{nF} / \mathrm{m}$ & 0.0354 & 0.0903 \\
\hline
\end{tabular}

\section{Boundary Conditions and Solution Procedure}

The finite element modeling was performed using COMSOL Multiphysics 5.0, wherein all the steps from model geometry creation to meshing, solution, and postprocessing were performed. The physics interface "piezoelectric devices" in the structural mechanics module of COMSOL Multiphysics was used. For three-dimensional models, tetrahedral elements are used for meshing. A direct solver, with a relative tolerance 
TABLE 3: Boundary conditions to evaluate the effective properties of the composite.

\begin{tabular}{|c|c|c|c|c|c|c|c|}
\hline Effective property & $B_{1}$ & $B_{2}$ & $B_{3}$ & $B_{4}$ & $B_{5}$ & $B_{6}$ & Formula \\
\hline$C_{11}^{\mathrm{eff}}, C_{12}^{\mathrm{eff}}$ & $\begin{array}{l}u=0 \\
\phi=0\end{array}$ & $\begin{array}{c}u=h \varepsilon_{0} \\
\phi=0\end{array}$ & $\begin{array}{l}v=0 \\
\phi=0\end{array}$ & $\begin{array}{l}v=0 \\
\phi=0\end{array}$ & $\begin{aligned} w & =0 \\
\phi & =0\end{aligned}$ & $\begin{aligned} w & =0 \\
\phi & =0\end{aligned}$ & $\begin{array}{l}C_{11}^{\mathrm{eff}}=\frac{\bar{\sigma}_{11}}{\bar{\varepsilon}_{11}} \\
C_{12}^{\mathrm{eff}}=\frac{\bar{\sigma}_{22}}{\bar{\varepsilon}_{11}}\end{array}$ \\
\hline$C_{13}^{\mathrm{eff}}, C_{33}^{\mathrm{eff}}$ & $\begin{array}{l}u=0 \\
\phi=0\end{array}$ & $\begin{array}{l}u=0 \\
\phi=0\end{array}$ & $\begin{array}{l}v=0 \\
\phi=0\end{array}$ & $\begin{array}{l}v=0 \\
\phi=0\end{array}$ & $\begin{aligned} w & =0 \\
\phi & =0\end{aligned}$ & $\begin{array}{c}w=h \varepsilon_{0} \\
\phi=0\end{array}$ & $\begin{array}{l}C_{13}^{\mathrm{eff}}=\frac{\bar{\sigma}_{11}}{\bar{\varepsilon}_{33}} \\
C_{33}^{\mathrm{eff}}=\frac{\bar{\sigma}_{33}}{\bar{\varepsilon}_{33}}\end{array}$ \\
\hline$C_{44}^{\mathrm{eff}}$ & $\begin{array}{l}u=0 \\
\phi=0\end{array}$ & $\begin{array}{l}u=0 \\
\phi=0\end{array}$ & $\begin{aligned} u & =0 \\
w & =0 \\
\phi & =0\end{aligned}$ & $\begin{aligned} u & =0 \\
w & =0 \\
\phi & =0\end{aligned}$ & $\begin{array}{l}u=0 \\
v=0 \\
\phi=0\end{array}$ & $\begin{array}{c}u=0 \\
v=2 h \varepsilon_{0} \\
\phi=0\end{array}$ & $C_{44}^{\mathrm{eff}}=\frac{\bar{\sigma}_{23}}{\left(2 \bar{\varepsilon}_{23}\right)}$ \\
\hline$C_{66}^{\mathrm{eff}}$ & $\begin{aligned} v & =0 \\
w & =0 \\
\phi & =0\end{aligned}$ & $\begin{aligned} v & =0 \\
w & =0 \\
\phi & =0\end{aligned}$ & $\begin{aligned} u & =0 \\
w & =0 \\
\phi & =0\end{aligned}$ & $\begin{array}{c}u=2 h \varepsilon_{0} \\
w=0 \\
\phi=0\end{array}$ & $\begin{aligned} w & =0 \\
\phi & =0\end{aligned}$ & $\begin{aligned} w & =0 \\
\phi & =0\end{aligned}$ & $C_{66}^{\mathrm{eff}}=\frac{\bar{\sigma}_{12}}{\left(2 \bar{\varepsilon}_{12}\right)}$ \\
\hline$\kappa_{11}^{\mathrm{eff}}$ & $\begin{array}{l}u=0 \\
\phi=0\end{array}$ & $\begin{array}{c}u=0 \\
\phi=V_{0}\end{array}$ & $v=0$ & $v=0$ & $w=0$ & $w=0$ & $\kappa_{11}^{\text {eff }}=\frac{\bar{D}_{1}}{\bar{E}_{1}}$ \\
\hline$e_{13}^{\mathrm{eff}}, e_{33}^{\mathrm{eff}}, \kappa_{33}^{\mathrm{efff}}$ & $u=0$ & $u=0$ & $v=0$ & $v=0$ & $\begin{aligned} w & =0 \\
\phi & =0\end{aligned}$ & $\begin{array}{l}w=0 \\
\phi=V_{0}\end{array}$ & $\begin{array}{c}e_{13}^{\text {eff }}=-\frac{\bar{\sigma}_{11}}{\bar{E}_{3}} \\
e_{33}^{\text {eff }}=-\frac{\bar{\sigma}_{33}}{\bar{E}_{3}} \\
\kappa_{33}^{\text {eff }}=\frac{\bar{D}_{3}}{\bar{E}_{3}}\end{array}$ \\
\hline$e_{15}^{\text {eff }}$ & $u=0$ & $u=0$ & $\begin{array}{l}v=0 \\
\phi=0\end{array}$ & $\begin{aligned} v & =0 \\
\phi & =V_{0}\end{aligned}$ & $w=0$ & $w=0$ & $e_{15}^{\mathrm{eff}}=-\frac{\bar{\sigma}_{23}}{\bar{E}_{2}}$ \\
\hline$Y_{3}^{\mathrm{eff}}, v_{31}^{\mathrm{eff}}$ & $\begin{array}{l}u=0 \\
\phi=0\end{array}$ & $\begin{array}{c}u=h \bar{\varepsilon}_{11} \\
\phi=0\end{array}$ & $\begin{array}{l}v=0 \\
\phi=0\end{array}$ & $\begin{array}{c}v=h \bar{\varepsilon}_{22} \\
\phi=0\end{array}$ & $\begin{aligned} w & =0 \\
\phi & =0\end{aligned}$ & $\begin{array}{c}w=h \varepsilon_{0} \\
\phi=0\end{array}$ & $\begin{array}{c}Y_{3}^{\mathrm{eff}}=\frac{\bar{\sigma}_{33}}{\bar{\varepsilon}_{33}} \\
v_{31}^{\mathrm{eff}}=-\frac{\bar{\varepsilon}_{11}}{\bar{\varepsilon}_{33}}\end{array}$ \\
\hline$Y_{2}^{\text {eff }}, v_{23}^{\text {eff }}$ & $\begin{array}{l}u=0 \\
\phi=0\end{array}$ & $\begin{array}{c}u=h \bar{\varepsilon}_{11} \\
\phi=0\end{array}$ & $\begin{array}{l}v=0 \\
\phi=0\end{array}$ & $\begin{array}{c}v=h \varepsilon_{0} \\
\phi=0\end{array}$ & $\begin{aligned} w & =0 \\
\phi & =0\end{aligned}$ & $\begin{array}{c}w=h \bar{\varepsilon}_{33} \\
\phi=0\end{array}$ & $\begin{array}{c}Y_{2}^{\mathrm{eff}}=\frac{\bar{\sigma}_{22}}{\bar{\varepsilon}_{22}} \\
v_{23}^{\mathrm{eff}}=-\frac{\bar{\varepsilon}_{33}}{\bar{\varepsilon}_{22}}\end{array}$ \\
\hline
\end{tabular}

of 0.001 , was used. The boundary conditions applied on the six surfaces of the cube are in the form of prescribed displacements and prescribed electric potentials. Boundary conditions applied and postprocessing steps for evaluating the effective electroelastic constants are presented in Table 3. In addition, the boundary conditions for evaluating some of the commonly used elastic properties like axial modulus $\left(Y_{2}^{\text {eff }}\right)$, transverse modulus $\left(Y_{3}^{\text {eff }}\right)$, and Poisson's ratios are also described. In Table 3, $u, v$, and $w$ are the displacement components along the $x$-, $y$-, and $z$-coordinate axes, respectively. $\varepsilon_{0}$ and $V_{0}$ are the applied strain and applied electric potential, respectively.

It is important to note that the finite element model and the associated boundary conditions presented in this section can only be applied to symmetric RVEs. These boundary conditions cannot be applied to evaluate effective properties of a composite considering nonsymmetric RVEs, such as those with completely random geometries and random arrangement of reinforcements. Another assumption implicit in this analysis is that the interface between $\mathrm{SU} 8$ and $\mathrm{ZnO}$ is perfect.

\section{Results}

In this section, effective material properties of a SU8 photoresist, reinforced with vertically aligned $\mathrm{ZnO}$ nanowires, are presented as a function of volume fraction of the $\mathrm{ZnO}$ nanowires. The volume fraction of $\mathrm{ZnO}$ nanowires ranges from zero to a maximum of 0.7. The volume fraction of the nanowires is increased by increasing the radius of the cylindrical nanowires, while keeping the length and number density of the nanowires constant. The effective material properties are evaluated as a function of $\mathrm{ZnO}$ volume fraction for all six types of RVEs described in Section 3 using the FEM and the analytical EMT micromechanics-based approach. Calculated data based on EMT appears in each plot as a solid curve or line.

Figure 2 shows that all components of the stiffness matrix exhibit a monotonic increase with increasing $\mathrm{ZnO}$ volume 

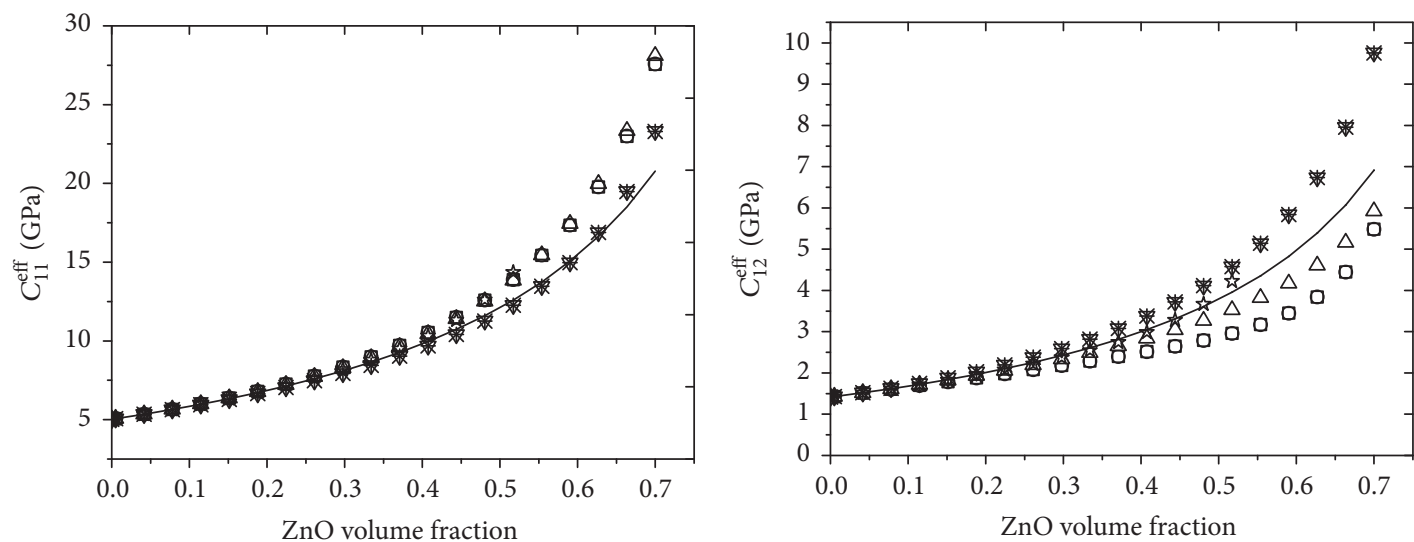

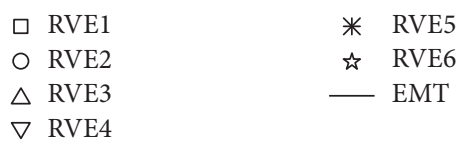

(a)

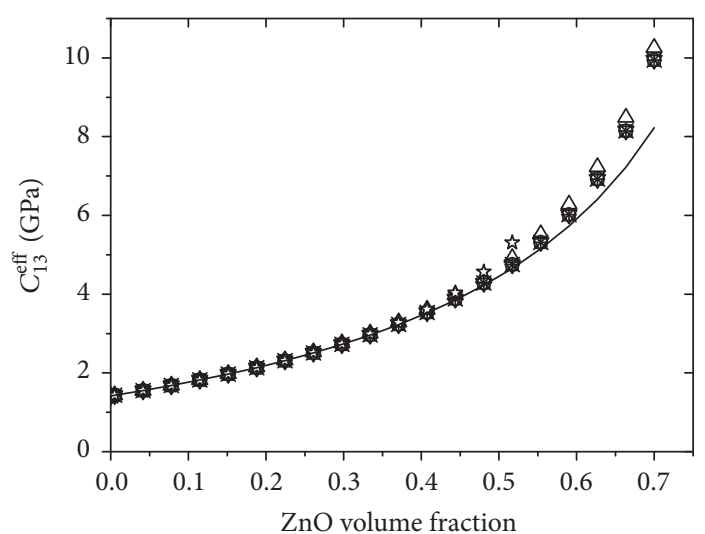
$\square$ RVE1
O RVE2
$\triangle$ RVE3

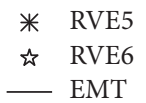

$\nabla$ RVE4

(c)

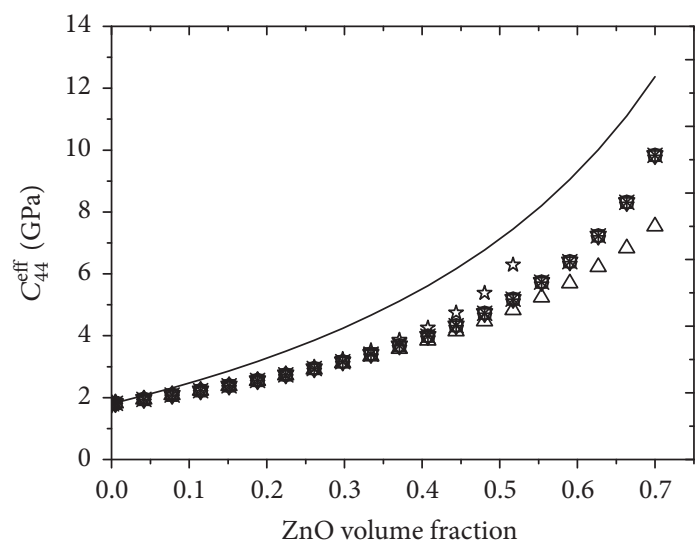

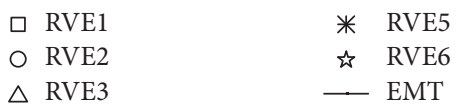

(b)

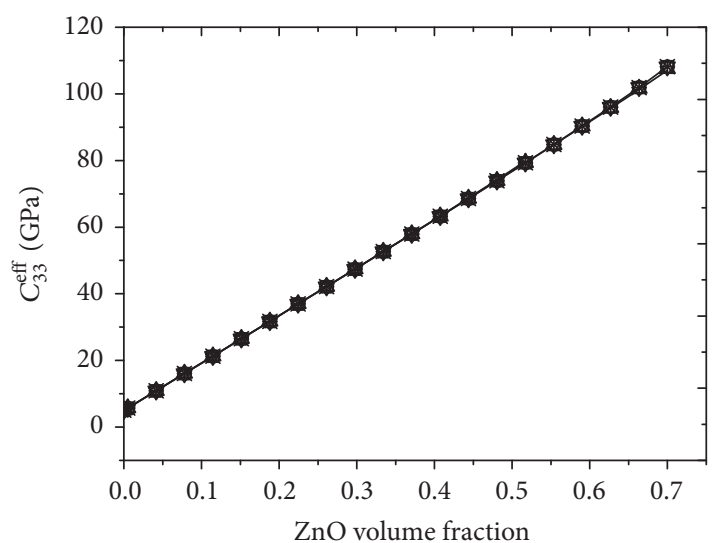

(d)

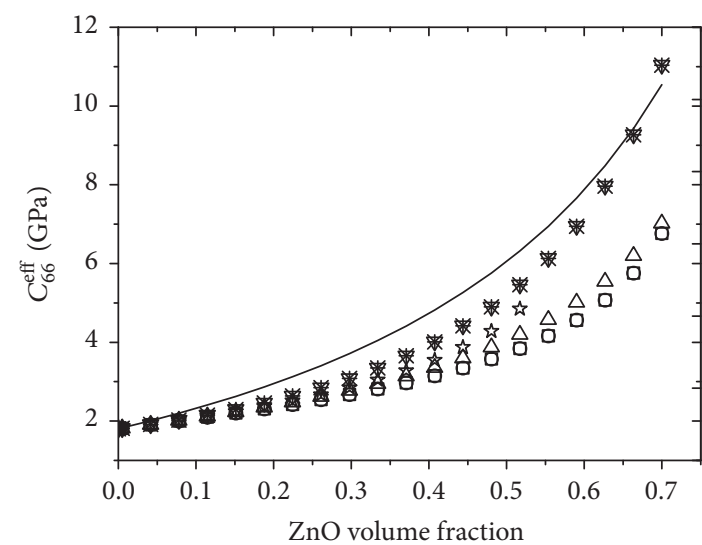
口 RVE1
O RVE2
$\triangle$ RVE3
* RVE5
« RVE6

$\nabla$ RVE4

(f)

Figure 2: Coefficients of the effective elastic stiffness matrix of SU8/ZnO unidirectional composite as a function of $\mathrm{ZnO}$ volume fraction: (a) $C_{11}^{\text {eff }}$, (b) $C_{12}^{\text {eff }}$, (c) $C_{13}^{\text {eff }}$, (d) $C_{33}^{\text {eff }}$, (e) $C_{44}^{\text {eff }}$, and (f) $C_{66}^{\text {eff }}$. 


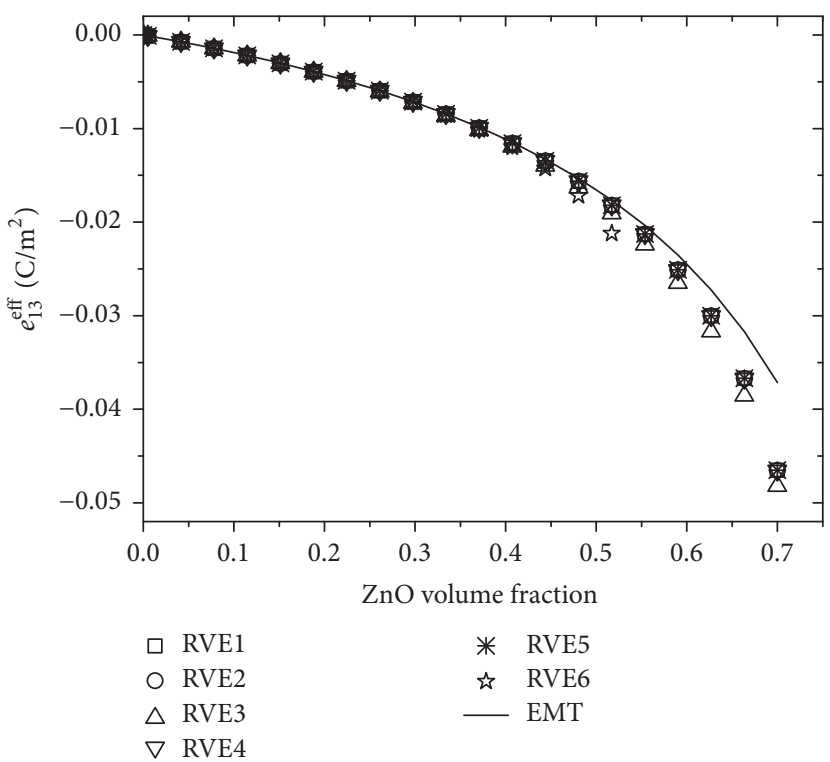

(a)

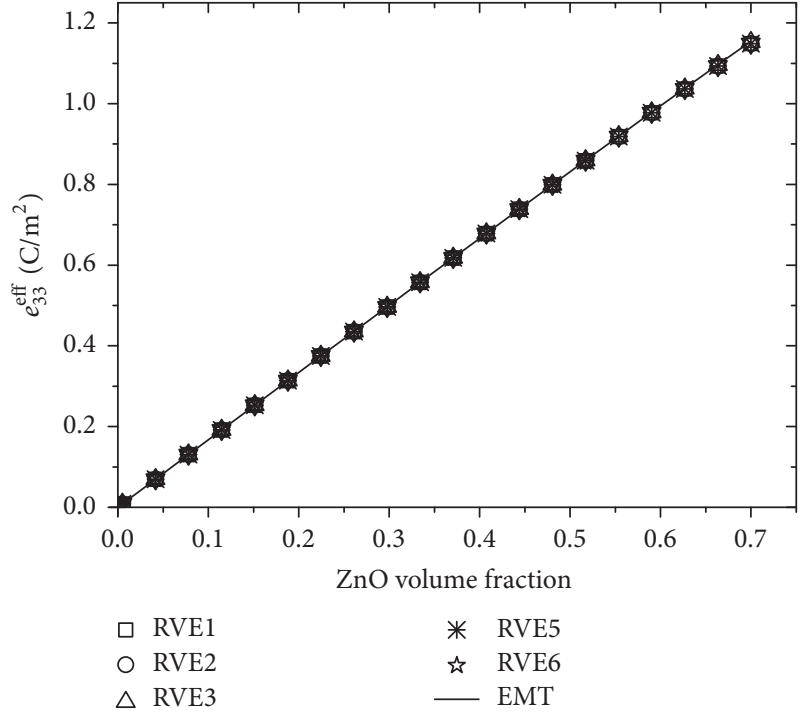

(b)

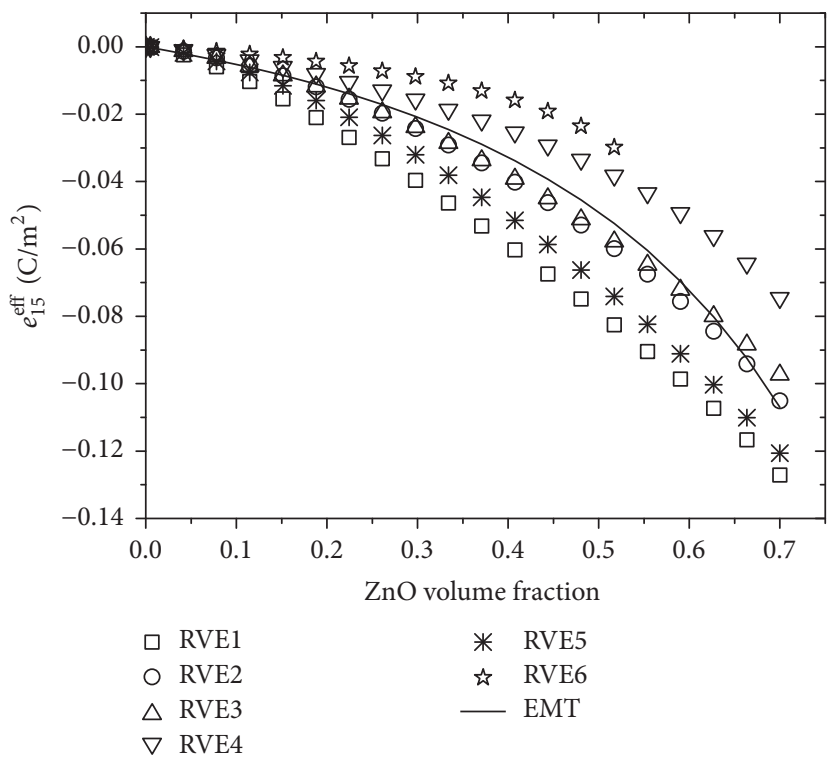

(c)

FIGURE 3: Coefficients of the effective piezoelectric modulus matrix of SU8/ZnO unidirectional composite as a function of $\mathrm{ZnO}$ volume fraction: (a) $e_{13}^{\text {eff }}$, (b) $e_{33}^{\text {eff }}$, and (c) $e_{15}^{\text {eff }}$.

fraction. In addition, it is observed that the doubling the size of the RVE does not have any effect on the effective mechanical properties. For example, the values obtained for effective mechanical properties for RVE1 and RVE2 coincide. Similarly, the effective mechanical properties obtained for RVE4 and for RVE5 are identical. It is also observed that values of coefficients of effective stiffness matrix obtained from the FEM match very well with the values obtained from the EMT-based approach at low volume fractions and in the case of $C_{33}^{\text {eff }}$ at all volume fractions.

From Figure 2(a), it is observed that predictions of $C_{11}^{\text {eff }}$ from EMT approach match best the results of FEM derived for RVE4 and for RVE5. From Figure 2(b), it can be seen that the values of $C_{12}^{\text {eff }}$ predicted by EMT-based approach match most closely the values obtained for RVE6 up to volume fraction of $\sim 0.5$. Figure 2 (c) shows that $C_{13}^{\text {eff }}$ is less structuresensitive compared to $C_{11}^{\text {eff }}$ and $C_{12}^{\text {eff }}$. EMT-based approach predicts lower values of $C_{13}^{\text {eff }}$ compared to those predicted by RVE-based finite element approach, especially at $\mathrm{ZnO}$ volume fractions higher than $\sim 0.5$. Close match between EMT-based approach and FEM approach is obtained for $C_{13}^{\text {eff }}$ for RVE1, REV2, RVE4, and RVE5. Figure 2(d) shows that $C_{33}^{\text {eff }}$ exhibits the least structure sensitivity among the coefficients 


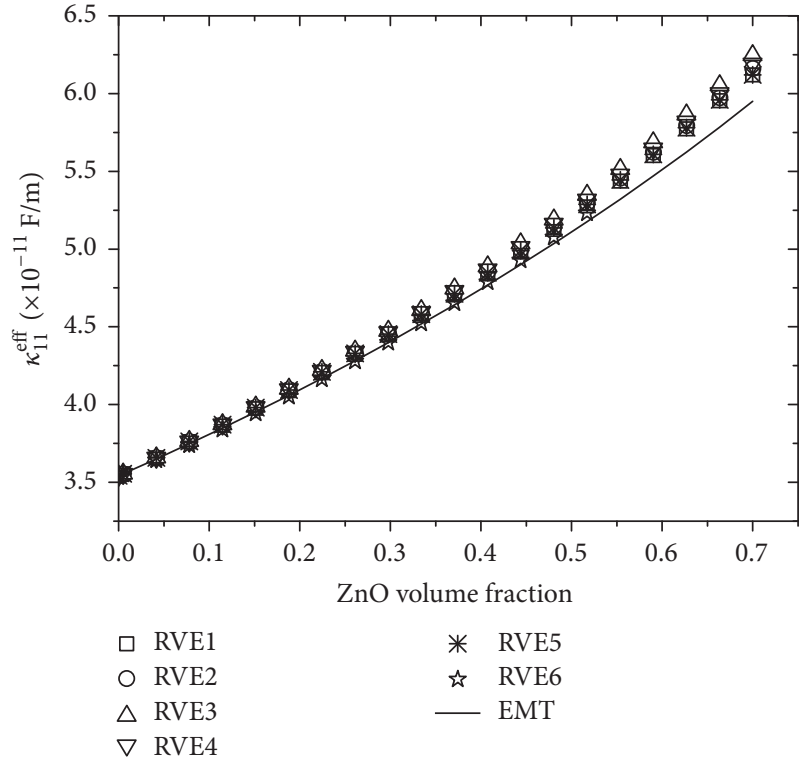

(a)

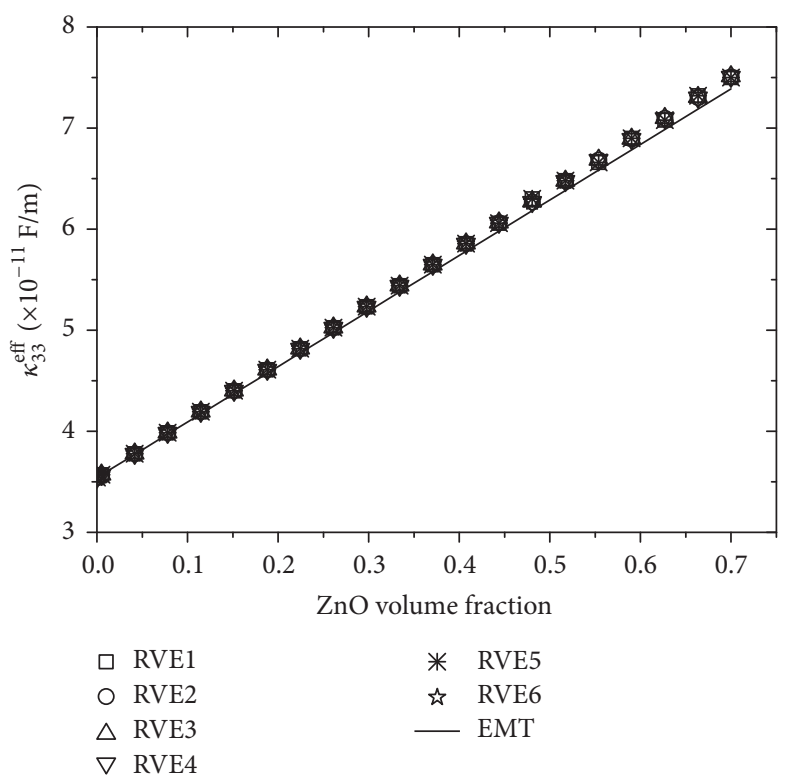

(b)

FIgURE 4: Coefficients of the effective dielectric modulus matrix of SU8/ZnO unidirectional composite as a function of $\mathrm{ZnO}$ volume fraction: (a) $\kappa_{11}^{\text {eff }}$ and (b) $\kappa_{33}^{\text {eff }}$.

of elastic stiffness matrix. The predicted values of all RVEs match with each other and with the values predicted by EMTbased approach. Figures 2(e) and 2(f) show that, for both $C_{44}^{\text {eff }}$ and $C_{66}^{\text {eff }}$, EMT-based approach predicts values which are greater than those predicted by FEM approach. For $C_{44}^{\text {eff }}$, the arrangement of the $\mathrm{ZnO}$ nanowires in square array and in face-centred square array produces similar values. Values of $C_{44}^{\text {eff }}$ obtained for RVE6 most closely match the values obtained from EMT approach. For $C_{66}^{\text {eff }}$, the values obtained for RVE4 and for RVE5 are in closest agreement with the values predicted by EMT approach.

Figure 3 shows the predicted values of the coefficients of the piezoelectric modulus matrix; $e_{13}^{\text {eff }}$ and $e_{15}^{\text {eff }}$ decrease while $e_{33}^{\text {eff }}$ increases monotonically with increasing volume fraction of $\mathrm{ZnO}$ nanowires. $e_{33}^{\text {eff }}$ is least sensitive to the arrangement of $\mathrm{ZnO}$ nanowires, while $e_{15}^{\text {eff }}$ is most sensitive to arrangement of the $\mathrm{ZnO}$ nanowires. From Figure 3(a), it is observed that predictions of $e_{13}^{\text {eff }}$ from the EMT-based approach match most closely the predictions from FEM for RVE4 and RVE5.

In case of $e_{13}^{\text {eff }}$, the values for RVEs with square array match the predictions of RVEs with face-centred square array. Figure 3(b) shows that predictions from EMT-based approach match the predictions of the values of $e_{33}^{\text {eff }}$ from FEM. Figure 3(c) shows that the values of $e_{15}^{\text {eff }}$ predicted by EMT-based approach are close to the values predicted for RVE2 and for RVE3.

Figure 4 shows that the nonzero, independent components of the effective dielectric modulus matrix, namely, $\kappa_{13}^{\text {eff }}$ and $\kappa_{33}^{\text {eff }}$, increase with increasing volume fraction of $\mathrm{ZnO}$ nanowires. These coefficients are not affected strongly by the arrangement of the nanowires, and predictions of the values of these coefficients by EMT-based approach match closely the values predicted by FEM.

The components of the effective piezoelectric strain coefficient matrix, $d_{31}^{\text {eff }}, d_{33}^{\text {eff }}$, and $d_{15}^{\text {eff }}$, are calculated using (15), (16), and (17), respectively. $d_{31}^{\text {eff }}$ decreases and $d_{33}^{\text {eff }}$ increases with increasing volume fraction of $\mathrm{ZnO}$ nanowires, as shown in Figures 5(a) and 5(b), respectively. These components are not sensitive to the arrangement of the nanowires and also match the predictions based on EMT approach. In contrast, as shown in Figure 5(c), $d_{15}^{\text {eff }}$ is found to be extremely sensitive to the arrangement of the $\mathrm{ZnO}$ nanowires and shows a complex behaviour on increasing volume fraction of $\mathrm{ZnO}$, where the value of $d_{15}^{\text {eff }}$ first decreases, then reaching a minimum for the $\mathrm{ZnO}$ volume fraction of $0.5-0.6$; thereafter, it finally increases continually up to the maximum $\mathrm{ZnO}$ volume fraction tested. The values of $d_{15}^{\text {eff }}$ predicted by the approach based on EMT method best match the predictions for RVE4.

Figure 6 shows that both axial and transverse moduli increase monotonically with increasing volume fraction of $\mathrm{ZnO}$. The axial modulus is not sensitive to the arrangement of the $\mathrm{ZnO}$ nanowires, and the predictions from EMT-based approach match the values predicted using the FEM. The transverse modulus, on the other hand, is more sensitive to the arrangement of nanowires, and the values obtained using the EMT approach most closely match the values predicted for RVE3.

Figure $7(a)$ shows that Poisson's ratio $v_{23}^{\text {eff }}$ decreases rapidly on increasing $\mathrm{ZnO}$ volume fraction, reaches a minimum at $\mathrm{ZnO}$ volume fraction of $\sim 0.3-0.4$, depending on the 


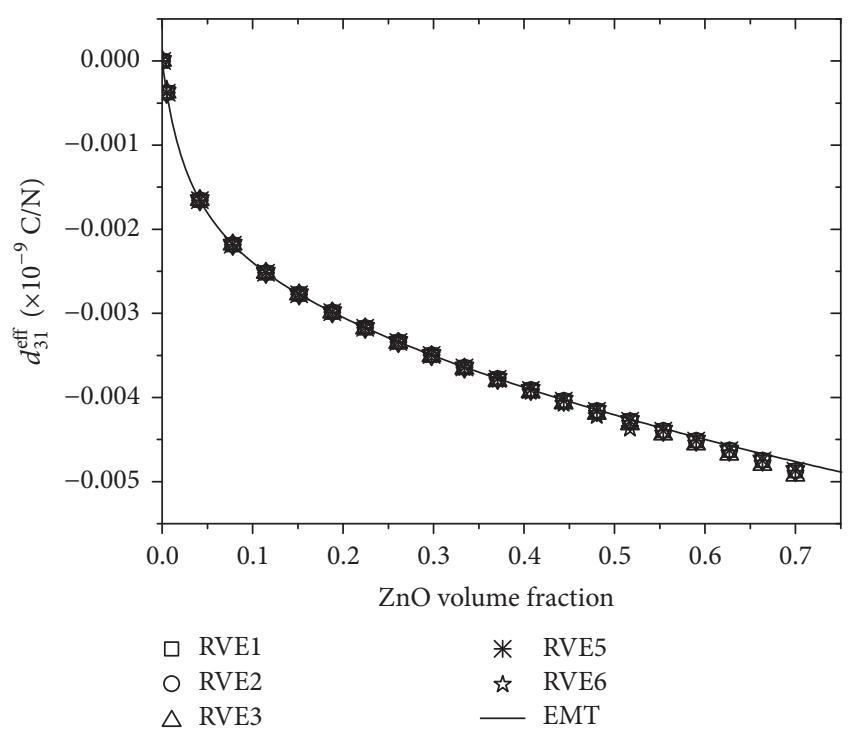

(a)

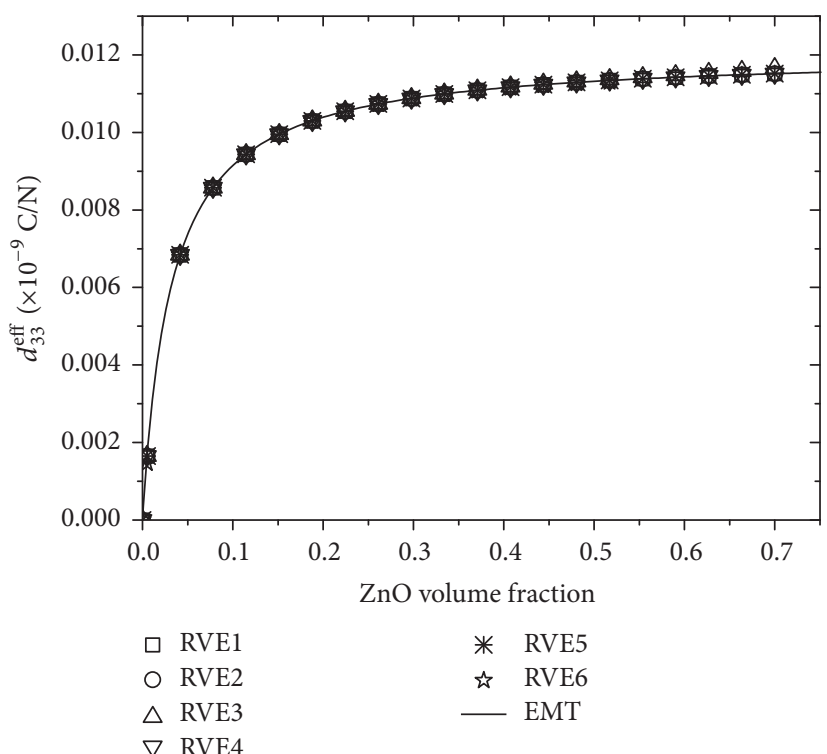

(b)

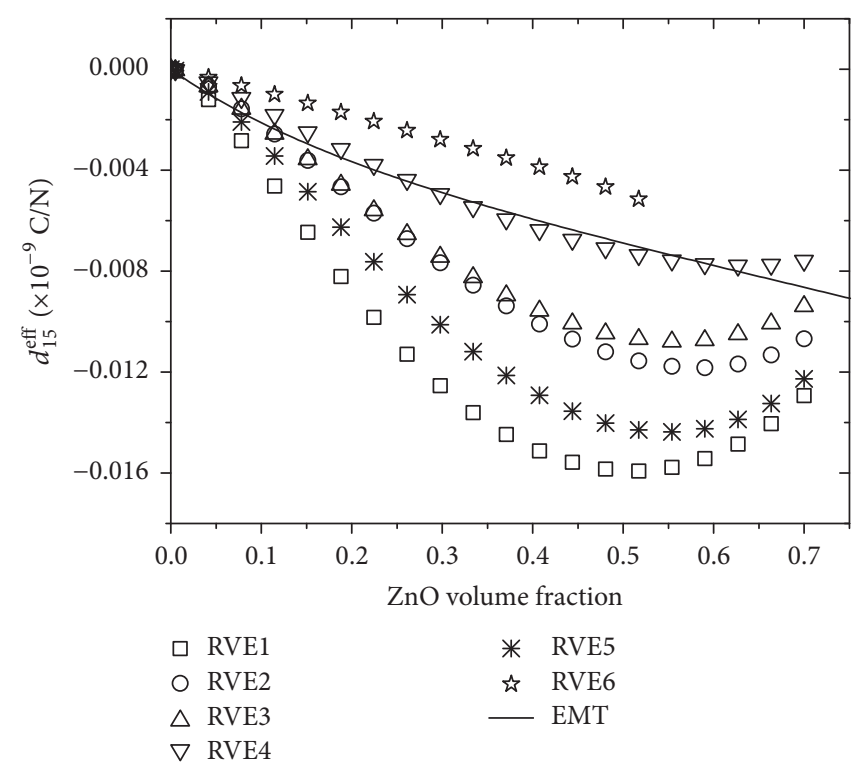

(c)

FIGURE 5: Components of the effective piezoelectric strain coefficient matrix of SU8/ZnO unidirectional composite as a function of ZnO volume fraction: (a) $d_{13}^{\text {eff }}$, (b) $d_{33}^{\text {eff }}$, and (c) $d_{15}^{\text {eff }}$.

RVE type, and then increases as volume fraction increases. RVE1 and RVE2 show similar values of $v_{23}^{\text {eff }}$, while RVE3, RVE4, and RVE5 match closely with each other and with the values predicted by an approach based on EMT method. RVE6 with quasi-random arrangement of nanowires generates slightly higher value of $v_{23}^{\text {eff }}$, compared to other RVEs.

Poisson's ratio, $v_{31}^{\text {eff }}$, increases monotonically from a value of 0.22 for pristine SU8 to a value of $\sim 0.30$ as the $\mathrm{ZnO}$ volume fraction increases to 0.7 , as shown in Figure 7 (b). The predicted values of $v_{31}^{\text {eff }}$ do not depend significantly on the arrangement of the $\mathrm{ZnO}$ nanowires. EMT-based approach underestimates $v_{31}^{\text {eff }}$ for $\mathrm{ZnO}$ volume fractions greater than
0.4. Close match between the two approaches is obtained for RVE1, RVE2, RVE3, and RVE4.

\section{Discussions}

The trends observed in this work for variation of effective material properties as a function of volume fraction are consistent with trends observed in other finite element analyses [25] and micromechanics-based analyses [19] for similar piezoelectric composites. All coefficients of the electroelastic matrix, as well as the derived material properties for the composite, are found to have values that are intermediate 


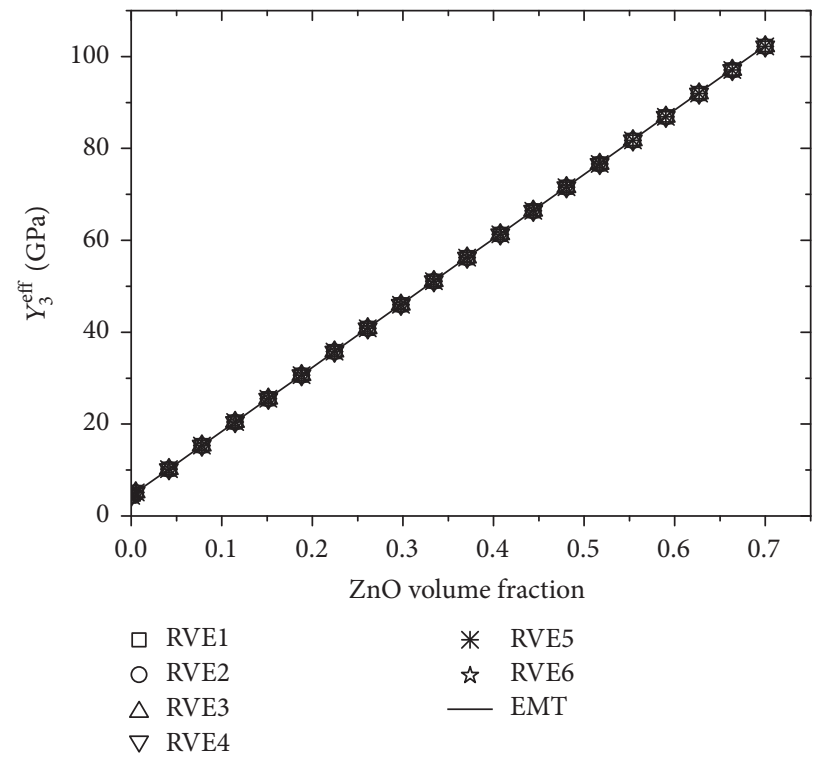

(a)

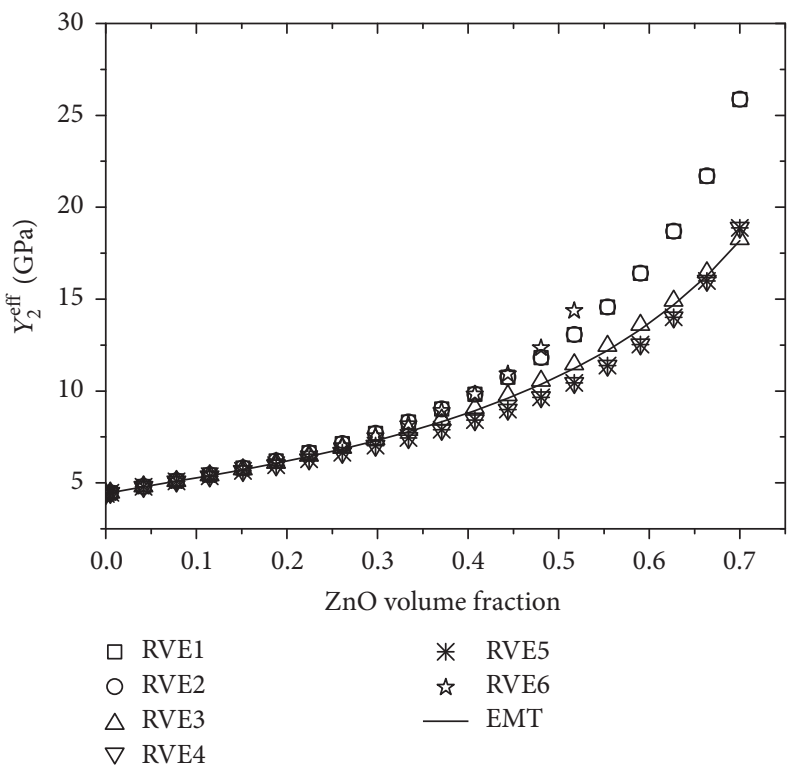

(b)

FIGURE 6: Effective elastic moduli of SU8/ZnO unidirectional composite as a function of $\mathrm{ZnO}$ volume fraction: (a) axial modulus ( $\left.Y_{3}^{\text {eff }}\right)$ and (b) transverse modulus $\left(Y_{2}^{\text {eff }}\right)$.

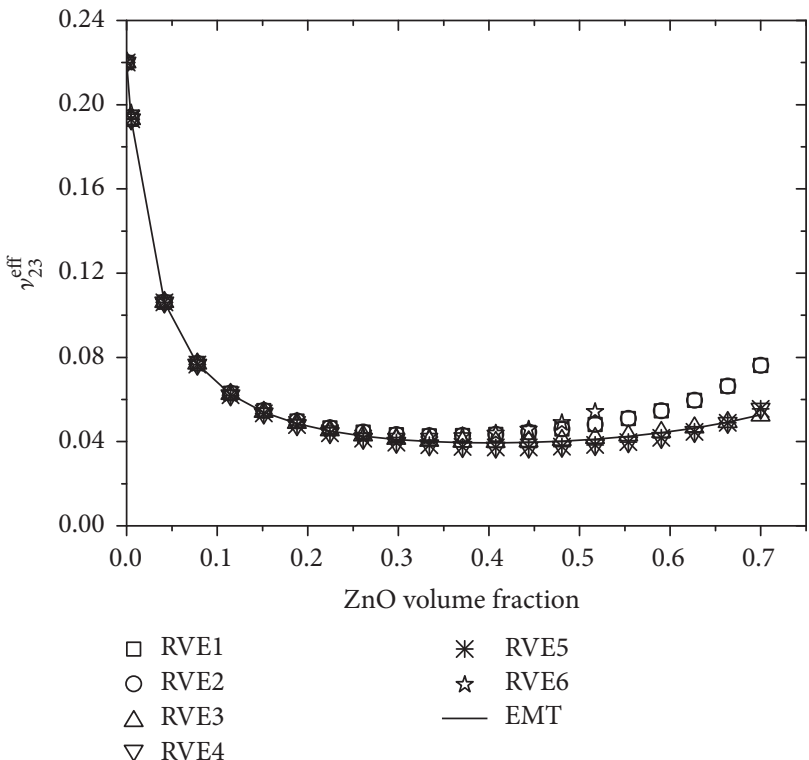

(a)

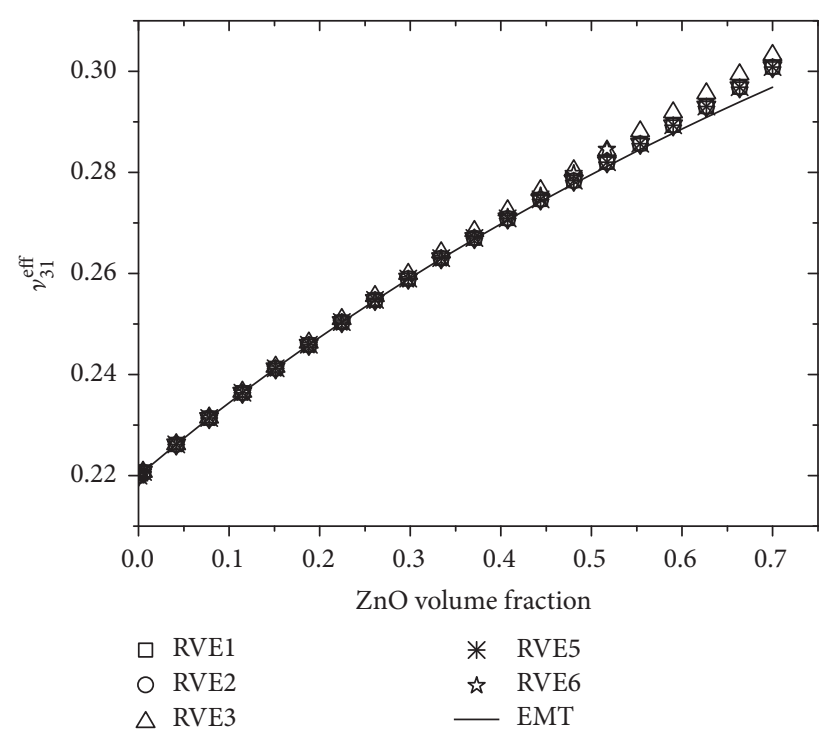

(b)

FIgURE 7: Effective Poisson's ratio of SU8/ZnO unidirectional composite as a function of $\mathrm{ZnO}$ volume fraction: (a) $v_{23}^{\text {eff }}$ and (b) $v_{31}^{\text {eff }}$.

between the values of SU8 and $\mathrm{ZnO}$. As the volume fraction of $\mathrm{ZnO}$ increases, the values of the coefficients change and tend to get closer to the value of the reinforcement phase. This change happens in a monotonic fashion. Experimental data on elastic, dielectric, and piezoelectric properties SU8/ZnO nanowire nanocomposites are not available for comparison with the predictions from FEM.

The observation that $e_{15}^{\text {eff }}$ has a strong dependence on the arrangement of the reinforcement phase is corroborated by
Berger et al. [53] and by Moreno et al. [42], where significant differences in values of $e_{15}^{\text {eff }}$ were reported when comparing reinforcement arrangements in square array and in hexagonal array. The reason for this strong dependence of $e_{15}^{\text {eff }}$ on arrangement is not understood. Analytical/semianalytical techniques, for example, the one based on EMT micromechanics presented in this study, are insensitive to arrangement of the reinforcement phase. A comparison of the results from a semianalytical method with the results obtained from FEM 
for different RVEs sheds light on the range of volume fractions, especially at low concentrations of $\mathrm{ZnO}$, where computationally simpler analytical/semianalytical techniques can be used without sacrificing accuracy.

\section{Conclusions}

Finite element method was used to evaluate effective elastic, piezoelectric, and dielectric properties of SU8 photoresist reinforced with vertically aligned $\mathrm{ZnO}$ nanowires, as a function of volume fraction of $\mathrm{ZnO}$ nanowires, up to a volume fraction of 0.7 for all RVEs except the one with quasi-random arrangement of nanowires, where the maximum volume fraction is $\sim 0.5$. Effective material properties like axial and transverse elastic moduli, Poisson's ratios, and components of the effective piezoelectric strain coefficient matrix were also derived for the SU8/ZnO nanocomposite. The values obtained from FEM for the effective properties are in close agreement with values obtained through a semianalytical EMT method, especially at low volume fractions of $\mathrm{ZnO}$.

Four different arrangements of $\mathrm{ZnO}$ nanowires were studied. The arrangement of the $\mathrm{ZnO}$ nanowires leads to differences in values obtained for transverse effective elastic and piezoelectric coefficients. However, these arrangements did not lead to significant differences in the effective axial/longitudinal properties of the composite.

For RVEs with square array and with face-centred square array of arrangement of $\mathrm{ZnO}$ nanowires, two sizes of RVE were studied. Doubling the size of the RVE did not have any significant effect on the coupled electromechanical properties of the piezoelectric composite, except for $e_{15}^{\text {eff }}$.

These results represent the first step towards design of efficient nanogenerators using polymer nanocomposites. The methodology presented in this work can be extended to studies that take into consideration the complex microstructural and interfacial features of the nanocomposite.

\section{Conflicts of Interest}

The authors declare that they have no conflicts of interest.

\section{Acknowledgments}

Financial support from the Department of Science and Technology-Science and Engineering Research Board (DSTSERB), Government of India, under Young Scientist Scheme (Grant no. YSS/2014/000830), and from Seed Grant, IIT Bhubaneswar (Grant no. SP062), is gratefully acknowledged.

\section{References}

[1] S. Lee, S. Bae, L. Lin et al., "Energy harvesting materials: superflexible nanogenerator for energy harvesting from gentle wind and as an active deformation sensor," Advanced Functional Materials, vol. 23, no. 19, pp. 2445-2449, 2013.

[2] X. D. Wang, "Piezoelectric nanogenerators-harvesting ambient mechanical energy at the nanometer scale," Nano Energy, vol. 1, no. 285, pp. 13-24, 2012.
[3] R. Yang, Y. Qin, C. Li, G. Zhu, and Z. L. Wang, "Converting biomechanical energy into electricity by a muscle-movementdriven nanogenerator," Nano Letters, vol. 9, no. 3, pp. 1201-1205, 2009.

[4] G. Zhu, R. Yang, S. Wang, and Z. L. Wang, "Flexible highoutput nanogenerator based on lateral $\mathrm{ZnO}$ nanowire array," Nano Letters, vol. 10, no. 8, pp. 3151-3155, 2010.

[5] Z. L. Wang and J. Song, "Piezoelectric nanogenerators based on zinc oxide nanowire arrays," Science, vol. 312, no. 5771, pp. $243-$ 246, 2006.

[6] Z. L. Wang, R. Yang, J. Zhou et al., "Lateral nanowire/nanobelt based nanogenerators, piezotronics and piezo-phototronics," Materials Science and Engineering R: Reports, vol. 70, no. 3-6, pp. 320-329, 2010.

[7] C.-Y. Chen, T.-H. Liu, Y. Zhou et al., "Electricity generation based on vertically aligned $\mathrm{PbZr}_{0.2} \mathrm{Ti}_{0.8} \mathrm{O}_{3}$ nanowire arrays," Nano Energy, vol. 1, no. 3, pp. 424-428, 2012.

[8] R. Hinchet, S. Lee, G. Ardila, L. Montès, M. Mouis, and Z. L. Wang, "Design and guideline rules for the performance improvement of vertically integrated nanogenerator," in Proceedings of PowerMEMS, pp. 38-41, Atlanta, Ga, USA, 2012.

[9] Y.-F. Lin, J. Song, Y. Ding, S.-Y. Lu, and Z. L. Wang, "Piezoelectric nanogenerator using CdS nanowires," Applied Physics Letters, vol. 92, Article ID 022105, pp. 2-5, 2008.

[10] A. Yu, H. Li, H. Tang, T. Liu, P. Jiang, and Z. L. Wang, "Vertically integrated nanogenerator based on $\mathrm{ZnO}$ nanowire arrays," Physica Status Solidi-Rapid Research Letters, vol. 5, no. 4, pp. 162-164, 2011.

[11] C. Oshman, C. Opoku, A. S. Dahiya, D. Alquier, N. Camara, and G. Poulin-Vittrant, "Measurement of Spurious Voltages in ZnO Piezoelectric Nanogenerators," Journal of Microelectromechanical Systems, vol. 25, no. 3, Article ID 7437376, pp. 533-541, 2016.

[12] R. F. Gibson, Principles of Composite Material Mechanics, CRC Press, Boca Raton, Fla, USA, Third edition, 2012.

[13] H. Banno, "Recent Developments of piezoelectric ceramic products and composites of synthetic rubber and piezoelectric ceramicparticles," Ferroelectrics, vol. 50, no. 1, pp. 3-12, 1983.

[14] P. Bisegna and R. Luciano, "Variational bounds for the overall properties of piezoelectric composites," Journal of the Mechanics and Physics of Solids, vol. 44, no. 4, pp. 583-602, 1996.

[15] Z. Hashin and S. Shtrikman, "A variational approach to the theory of the elastic behaviour of multiphase materials," Journal of the Mechanics and Physics of Solids, vol. 11, pp. 127-140, 1963.

[16] P. Bisegna and R. Luciano, "On methods for bounding the overall properties of periodic piezoelectric fibrous composites," Journal of the Mechanics and Physics of Solids, vol. 45, no. 8, pp. 1329-1356, 1997.

[17] J. D. Eshelby, "The determination of the elastic field of an ellipsoidal inclusion, and related problems," Proc R Soc A Math Phys Eng Sci, vol. 241, pp. 376-396, 1957.

[18] M. L. Dunn and M. Taya, "An analysis of piezoelectric composite materials containing ellipsoidal inhomogeneities," Proc R Soc A Math Phys Eng Sci, vol. 443, no. 1918, pp. 265-287, 1993.

[19] M. L. Dunn and M. Taya, "Micromechanics predictions of the effective electroelastic moduli of piezoelectric composites," International Journal of Solids and Structures, vol. 30, no. 2, pp. 161-175, 1993.

[20] Y. Benveniste, "The determination of the elastic and electric fields in a piezoelectric inhomogeneity," Journal of Applied Physics, vol. 72, no. 3, pp. 1086-1095, 1992. 
[21] T. Mori and K. Tanaka, "Average stress in matrix and average elastic energy of materials with misfitting inclusions," Acta Metallurgica, vol. 21, no. 5, pp. 571-574, 1973.

[22] Y. Benveniste, "A new approach to the application of MoriTanaka's theory in composite materials," Mechanics of Materials, vol. 6, no. 2, pp. 147-157, 1987.

[23] B. Budiansky, "On the elastic moduli of some heterogeneous materials," Journal of the Mechanics and Physics of Solids, vol. 13, no. 4, pp. 223-227, 1965.

[24] R. Mclaughlin, "A study of the differential scheme for composite materials," International Journal of Engineering Science, vol. 15, no. 4, pp. 237-244, 1977.

[25] G. M. Odegard, "Constitutive modeling of piezoelectric polymer composites," Acta Materialia, vol. 52, no. 18, pp. 5315-5330, 2004.

[26] R. Guinovart-Díaz, J. Bravo-Castillero, R. Rodríguez-Ramos, F. J. Sabina, and R. Martínez-Rosado, "Overall properties of piezocomposite materials 1-3," Materials Letters, vol. 48, no. 2, pp. 93-98, 2001.

[27] R. Singh, P. D. Lee, T. C. Lindley et al., "Characterization of the deformation behavior of intermediate porosity interconnected Ti foams using micro-computed tomography and direct finite element modeling," Acta Biomaterialia, vol. 6, no. 6, pp. 23422351, 2010.

[28] C. T. Sun and R. S. Vaidya, "Prediction of composite properties from a representative volume element," Composites Science and Technology, vol. 56, no. 2, pp. 171-179, 1996.

[29] P. Gaudenzi, "On the electromechanical response of active composite materials with piezoelectric inclusions," Computers and Structures, vol. 65, no. 2, pp. 157-168, 1997.

[30] C. Poizat and M. Sester, "Effective properties of composites with embedded piezoelectric fibres," Computational Materials Science, vol. 16, no. 1-4, pp. 89-97, 1999.

[31] H. E. Pettermann and S. Suresh, "A comprehensive unit cell model: a study of coupled effects in piezoelectric 1-3 composites," International Journal of Solids and Structures, vol. 37, no. 39, pp. 5447-5464, 2000.

[32] R. Kar-Gupta and T. A. Venkatesh, "Electromechanical response of 1-3 piezoelectric composites: a numerical model to assess the effects of fiber distribution," Acta Materialia, vol. 55, no. 4, pp. 1275-1292, 2007.

[33] R. Kar-Gupta and T. A. Venkatesh, "Electromechanical response of piezoelectric composites: Effects of geometric connectivity and grain size," Acta Materialia, vol. 56, no. 15, pp. 3810-3823, 2008.

[34] R. Kar-Gupta and T. A. Venkatesh, "Electromechanical response of 1-3 piezoelectric composites: effect of poling characteristics," Journal of Applied Physics, vol. 98, no. 5, Article ID 54102, 2005.

[35] H. Berger, S. Kari, U. Gabbert et al., "Unit cell models of piezoelectric fiber composites for numerical and analytical calculation of effective properties," Smart Materials and Structures, vol. 15, no. 2, pp. 451-458, 2006.

[36] H. Berger, S. Kari, U. Gabbert, R. Rodriguez-Ramos, J. BravoCastillero, and R. Guinovart-Diaz, "Calculation of effective coefficients for piezoelectric fiber composites based on a general numerical homogenization technique," Composite Structures, vol. 71, no. 3-4, pp. 397-400, 2005.

[37] R. d. Medeiros, M. E. Moreno, F. D. Marques, and V. Tita, "Effective properties evaluation for smart composite materials," Journal of the Brazilian Society of Mechanical Sciences and Engineering, vol. 34, no. spe, pp. 362-370, 2012.
[38] M. N. Rao, S. Tarun, R. Schmidt, and K.-U. Schröder, "Finite element modeling and analysis of piezo-integrated composite structures under large applied electric fields," Smart Materials and Structures, vol. 25, no. 5, Article ID 055044, 2016.

[39] M. C. Ray and A. K. Pradhan, "The performance of vertically reinforced 1-3 piezoelectric composites in active damping of smart structures," Smart Materials and Structures, vol. 15, no. 2, pp. 631-641, 2006.

[40] T. Ikeda, Fundamentals of Piezolectricity, Oxford University Press, Oxford, UK, 1990.

[41] D. M. Barnett and J. Lothe, "Dislocations and line charges in anisotropic piezoelectric insulators," Physica Status Solidi B, vol. 67, no. 1, pp. 105-111, 1975.

[42] M. E. Moreno, V. Tita, and F. D. Marques, "Influence of boundary conditions on the determination of effective material properties for active fiber composites," in Proceedings of the 11th Pan-American Congress of Applied Mechanics, Foz do Iguacu, PR, Brazil, 2010.

[43] J. Aboudi, Mechanics of Composite Materials: A Unified Micromechanical Approach, Elsevier Science Publishers, Amsterdam, The Netherlands, 1991.

[44] M. L. Dunn, "Electroelastic green's functions for transversely isotropic piezoelectric media and their application to the solution of inclusion and inhomogeneity problems," International Journal of Engineering Science, vol. 32, no. 1, pp. 119-131, 1994.

[45] F. Chollet, "SU-8: thick photo-resist for MEMS," 2013, http:// memscyclopedia.org/su8.html.

[46] V. Wang, Photoacoustic Imaging and Spectroscopy, CRC Press, Boca Raton, Fla, USA, First edition, 2009.

[47] C. Jagadish and S. J. Pearton, Eds., Zinc Oxide Bulk, Thin Films and Nanostructures: Processing, Properties, and Applications, Elsevier, Hong Kong, First edition, 2006.

[48] P. Ray, V. Seena, R. A. Khare, A. R. Bhattacharyya, P. R. Apte, and R. Rao, "SU8/modified MWNT composite for piezoresistive sensor application," Materials Research Society Proceedings, vol. 1299, pp. 135-140, 2011.

[49] V. Seena, A. Fernandes, P. Pant, S. Mukherji, and V. Ramgopal Rao, "Polymer nanocomposite nanomechanical cantilever sensors: material characterization, device development and application in explosive vapour detection," Nanotechnology, vol. 22, no. 29, Article ID 295501, 2011.

[50] K. Prashanthi, M. Naresh, V. Seena, T. Thundat, and V. Ramgopal Rao, "A novel photoplastic piezoelectric nanocomposite for MEMS applications," Journal of Microelectromechanical Systems, vol. 21, no. 2, Article ID 6118300, pp. 259-261, 2012.

[51] K. Prashanthi, N. Miriyala, R. D. Gaikwad, W. Moussa, V. R. Rao, and T. Thundat, "Vibtrational energy harvesting using photo-patternable piezoelectric nanocomposite cantilevers," Nano Energy, vol. 2, no. 5, pp. 923-932, 2013.

[52] M. Kandpal, C. Sharan, P. Poddar, K. Prashanthi, P. R. Apte, and V. Ramgopal Rao, "Photopatternable nano-composite (SU$8 / \mathrm{ZnO}$ ) thin films for piezo-electric applications," Applied Physics Letters, vol. 101, no. 10, Article ID 104102, 2012.

[53] H. Berger, S. Kari, U. Gabbert et al., "An analytical and numerical approach for calculating effective material coefficients of piezoelectric fiber composites," International Journal of Solids and Structures, vol. 42, no. 21-22, pp. 5692-5714, 2005. 

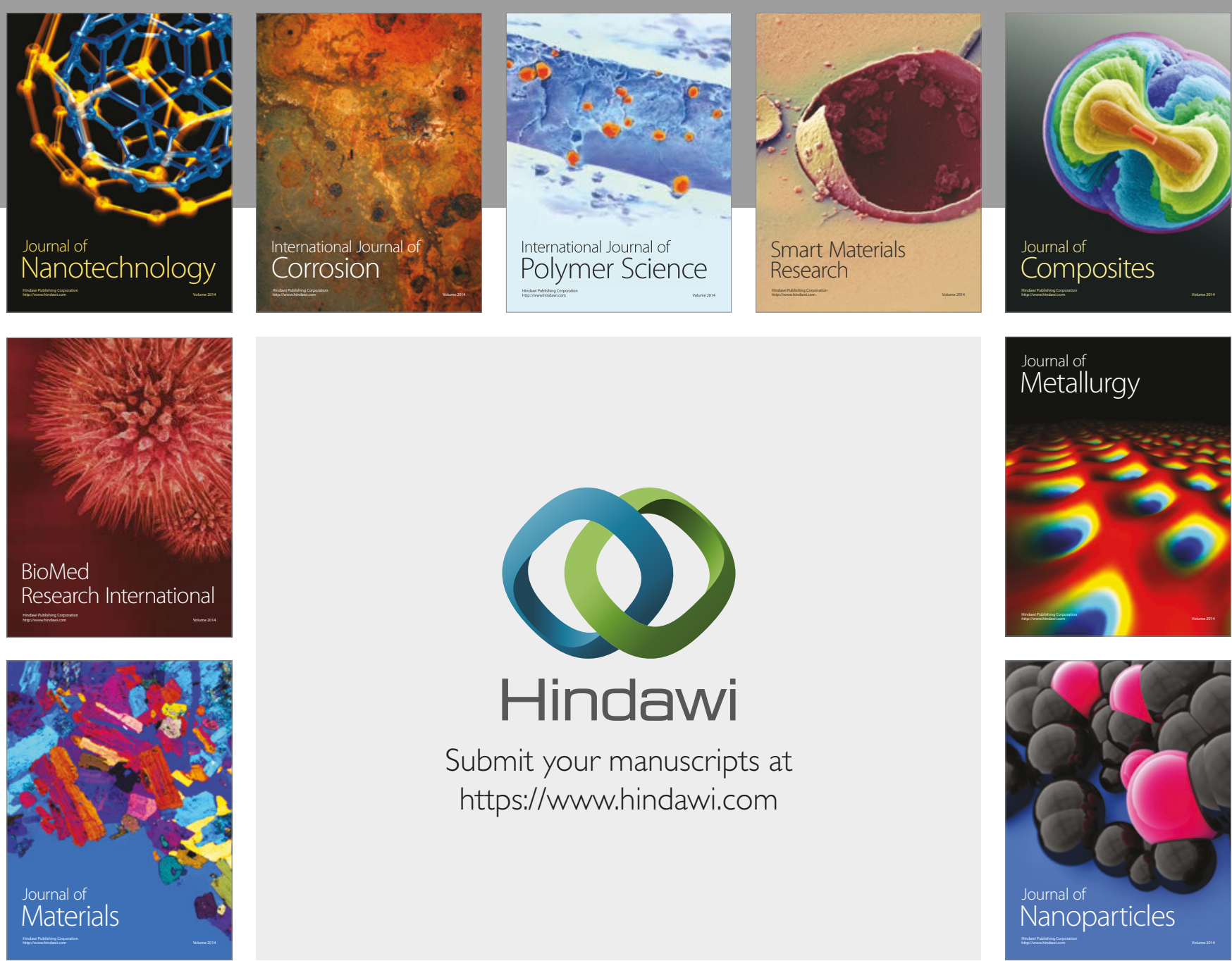

\section{Hindawi}

Submit your manuscripts at

https://www.hindawi.com
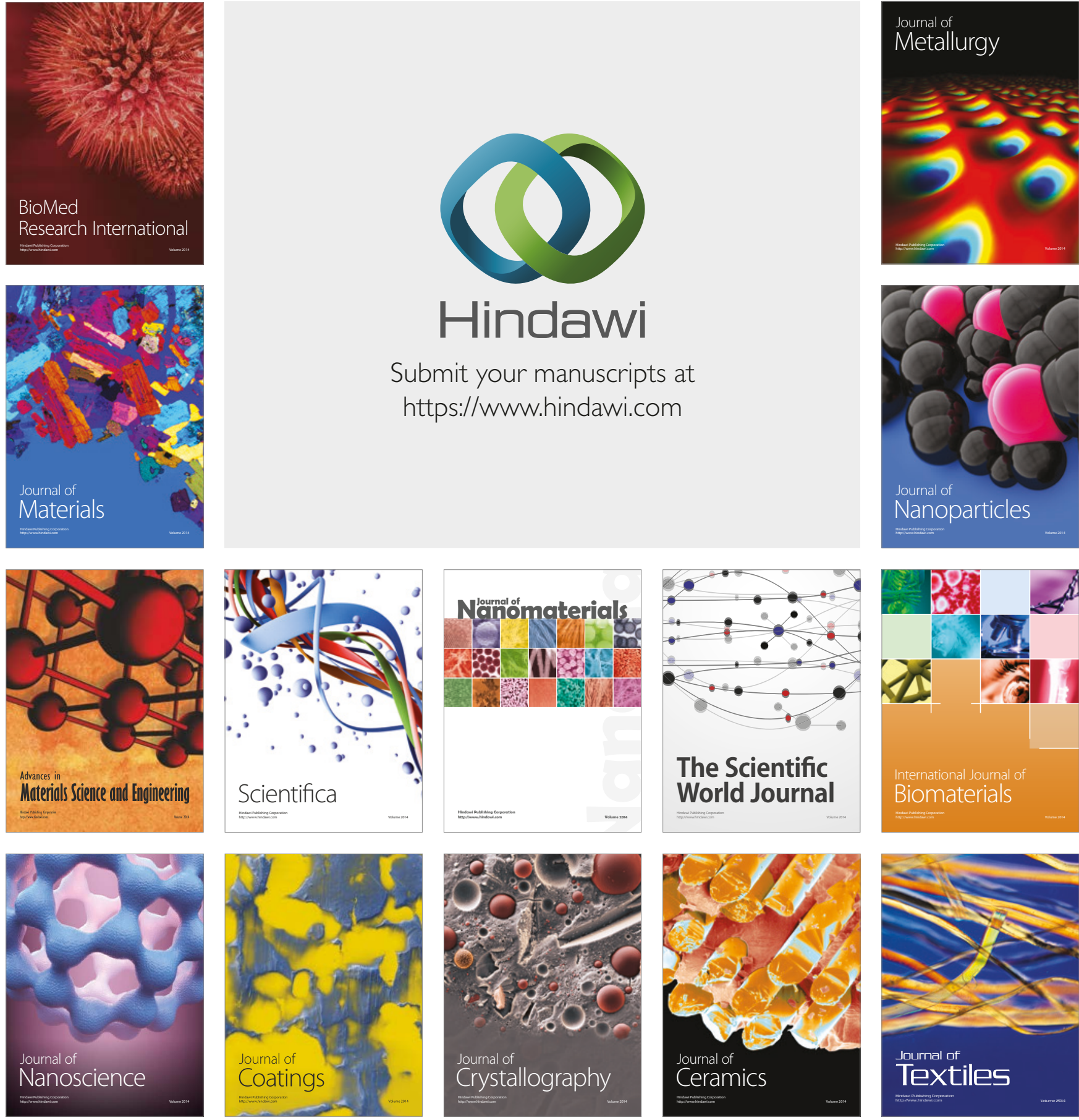

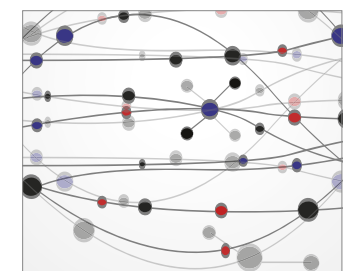

The Scientific World Journal
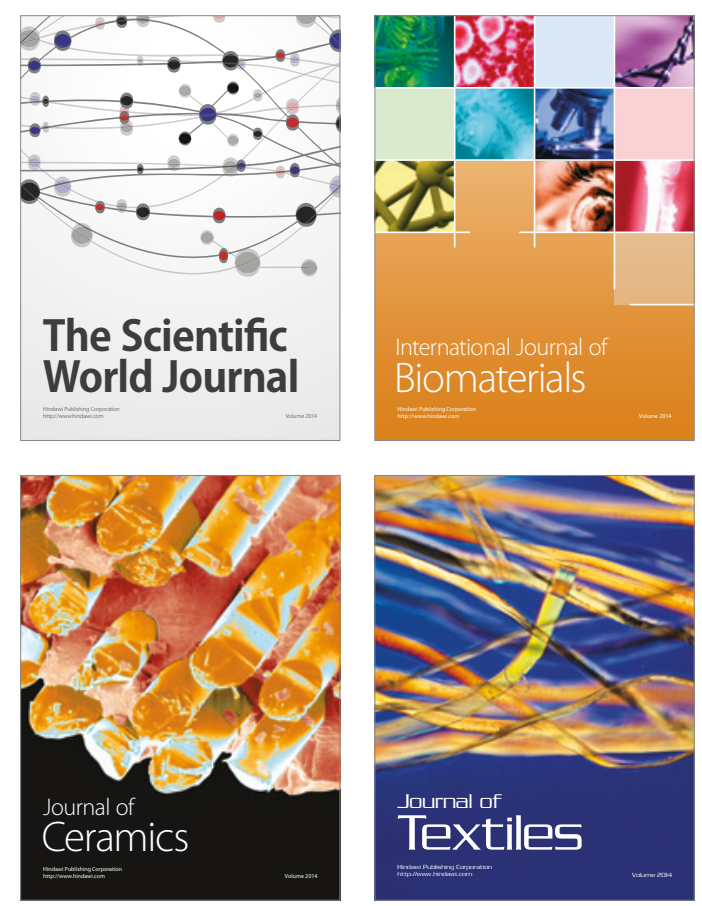